\title{
COLLAGEN TYPE X IS ESSENTIAL FOR SUCCESSFUL MESENCHYMAL STEM CELL-MEDIATED CARTILAGE FORMATION AND SUBSEQUENT ENDOCHONDRAL OSSIFICATION
}

\author{
C.A. Knuth ${ }^{1}$, E. Andres Sastre ${ }^{1,2}$, N.B. Fahy ${ }^{1,2}$, J. Witte-Bouma ${ }^{1}$, Y. Ridwan ${ }^{3,4}$, E.M. Strabbing ${ }^{1}$, \\ M.J. Koudstaal ${ }^{1}$, J. van de Peppel ${ }^{5}$, E.B. Wolvius ${ }^{1}$, R. Narcisi ${ }^{2}$ and E. Farrell ${ }^{1, *}$
}

\author{
${ }^{1}$ Department of Oral and Maxillofacial Surgery, Special Dental Care and Orthodontics, Erasmus MC, \\ University Medical Centre, 3015 CE Rotterdam, the Netherlands \\ ${ }^{2}$ Department of Orthopaedics, Erasmus MC, 3015 CE Rotterdam, the Netherland \\ ${ }^{3}$ Department of Molecular Genetics, University Medical Centre Rotterdam, Erasmus MC, \\ 3015 CE Rotterdam, the Netherlands \\ ${ }^{4}$ Department of Radiology and Nuclear Medicine, Erasmus MC, University Medical Centre Rotterdam, \\ 3015 CE Rotterdam, the Netherlands \\ ${ }^{5}$ Department of Internal Medicine, Erasmus MC, 3015 CE Rotterdam, the Netherlands
}

\begin{abstract}
In tissue engineering, endochondral ossification (EO) is often replicated by chondrogenically differentiating mesenchymal stromal cells (MSCs) in vitro and achieving bone formation through in vivo implantation. The resulting marrow-containing bone constructs are promising as a treatment for bone defects. However, limited bone formation capacity has prevented them from reaching their full potential. This is further complicated since it is not fully understood how this bone formation is achieved. Acellular grafts derived from chondrogenically differentiated MSCs can initiate bone formation; however, which component within these decellularised matrices contribute to bone formation has yet to be determined. Collagen type X(COLX), a hypertrophy-associated collagen found within these constructs, is involved in matrix organisation, calcium binding and matrix vesicle compartmentalisation. However, the importance of COLX during tissue-engineered chondrogenesis and subsequent bone formation is unknown. The present study investigated the importance of COLX by shRNA-mediated gene silencing in primary MSCs. A significant knock-down of COLX disrupted the production of extracellular matrix key components and the secretion profile of chondrogenically differentiated MSCs. Following in vivo implantation, disrupted bone formation in knock-down constructs was observed. The importance of COLX was confirmed during both chondrogenic differentiation and subsequent EO in this tissue engineering setting.
\end{abstract}

Keywords: Collagen type $X$, endochondral ossification, regenerative medicine, bone tissue engineering, mesenchymal stem cells, chondrogenesis.

*Address for correspondence: Dr Eric Farrell, Department of Oral and Maxillofacial Surgery, Special Dental Care and Orthodontics, Erasmus MC, 3000 DR Rotterdam, the Netherlands.

Telephone number: +31 107043655 Fax number: +31 107033098 Email: e.farrell@erasmusmc.nl

Copyright policy: This article is distributed in accordance with Creative Commons Attribution Licence (http://creativecommons.org/licenses/by-sa/4.0/).

\section{Introduction}

Endochondral ossification (EO) is the process by which long bones are formed. It relies on mesenchymal cells, which condense and undergo chondrogenic differentiation, resulting in the formation of a cartilage anlage (Ornitz, 2005). Subsequently, this anlage is remodelled by osteoclasts (Ortega et al., 2004), invaded by nearby vasculature (Maes et al., 2010) and, ultimately, serves as the template for bone formation by osteoblasts (Mackie, 2003). EO can be mimicked in a tissue engineering setting by chondrogenically differentiating mesenchymal stem cells (MSCs) pre-implantation (Farrell et al., 2011; Farrell et al., 2008; Scotti et al., 2013; Scotti et al., 2010; van der Stok et al., 2014). Although in the future such grafts could offer an attractive alternative to currently available treatment options, these constructs exhibit variable degrees of bone formation, with variable success in large-defect repair, depending on the approach used (Knuth et al., 2017; van der Stok et al., 2014). In recent years, the importance of donor 
cells in experimental models has been identified, as they are capable of directly contributing to bone formation. There is evidence suggesting that, during development (Cervantes-Diaz et al., 2017; Yang et al., 2014; Zhou et al., 2014), implanted cells can directly contribute to osteoblast-like cell populations (Bahney et al., 2014; Scotti et al., 2010). However, there is still much to learn about these grafts. Recently, it has been shown that devitalised grafts derived from chondrogenic MSCs form endochondral bone following in vivo implantation (Bourgine et al., 2014; Cunniffe et al., 2015), indicating that components within the chondrogenic matrix itself are capable of inducing bone formation independent of the presence of MSCs. Cunniffe et al. (2015) further showed that biomaterials containing decellularised matrices, specifically those originating from hypertrophic chondrogenically differentiated MSCs, are far better at inducing this bone formation than nonhypertrophic counterparts. However, what exactly within these hypertrophic matrices is responsible for this difference remains to be determined.

During chondrogenic differentiation, collagen type $X(C O L X)$, the major marker of hypertrophic chondrocytes, can be detected within the first few days (Knuth et al., 2017; Pelttari et al., 2006). Developmentally, following hypertrophic differentiation, the extracellular matrix (ECM) is remodelled to allow for invasion of the template by the nearby vasculature (Bahney et al., 2015; Colnot and Helms, 2001). Next, mineralisation of the cartilage template is initiated, osteoclastic remodelling occurs throughout the process of matrix remodelling, allowing for easier cell migration and vascularisation to occur (Colnot and Helms, 2001; Engsig et al., 2000; Touaitahuata et al., 2014), and, ultimately, endochondral bone formation takes place (Colnot and Helms, 2001; Deckers et al., 2002). COLX is produced by hypertrophic chondrocytes (HCs) and comprises more than $45 \%$ of the total collagens produced (Luvalle et al., 1992). It plays several important roles during EO, even contributing to the structural support of the pericellular network (Schmid and Linsenmayer, 1985; Shen, 2005), support that is essential during matrix remodelling (Shen, 2005). Additionally, COLX may play a role in initiating biomineralisation by binding annexin V (Kirsch and Pfäffle, 1992; Wu et al., 1991). Annexin V facilitates the influx of $\mathrm{Ca}^{2+}$ into matrix vesicles, initiating mineralisation (Anderson et al., 2005). Others have hypothesised that COLX is also essential for the proper distribution of collagens and proteoglycans throughout the ECM (Chan and Jacenko, 1998; Jacenko et al., 2001), further supporting a role of COLX in proper matrix organisation. However, these hypotheses have been hard to verify since previous studies utilising transgenic (Tg) mouse models in which COLX is removed, truncated or disrupted using different methods produce conflicting data (Campbell et al., 2004; Jacenko et al., 2001; Kim et al., 2003; Kwan et al.,
1997; Rosati et al., 1994). Some groups have shown early developmental irregularities attributed to a lack of COLX, including perinatal death, dwarfism and various skeletal abnormalities (Campbell et al., 2004; Jacenko et al., 2001). Rosati et al. (1994) reported a complete absence of phenotypic changes. Studies have also reported that a lack of COLX results in a compromised immune system, with an inability to elicit and control a proper immune reaction (Jacenko et al., 2002; Sweeney et al., 2008; Sweeney et al., 2010), while others find no such deficiency (Rosati et al., 1994). These different findings could come down to the genetic variation among different strains of mice used, variation in the methodology used to achieve knock-down/interference of COLX or differences in sample processing, analysis or staining techniques. Nevertheless, this makes understanding the role COLX increasingly challenging. Furthermore, COLX has only been studied from a developmental stand point. How COLX contributes to both chondrogenic differentiation of MSCs and subsequent endochondral bone formation has not been studied. The goal of the present study was to determine if COLX is essential for MSC-mediated chondrogenic differentiation and subsequent endochondral bone formation. COL10A1 expression was knocked-down using a lentivirusdelivered short hairpin RNA (shRNA) directed against COL10A1 mRNA sequence. In this way, it was possible to specifically knock-down the production of COLX in MSCs and determine its contribution to tissue engineered chondrogenesis and endochondral bone formation. COL10A1 knock-down resulted in disrupted chondrogenic differentiation. When the knock-down was highly efficient, the production of key ECM components was severely decreased. Several secreted factors linked to the recruitment of key cell types involved in EO were also downregulated. Following in vivo implantation, EO was likewise affected. However, this was only observed when knock-down efficiency was more than $80 \%$, indicating that a minimal production of COL10A1 was sufficient to prevent these adverse effects. The study showed the importance of COLX in MSCmediated chondrogenesis and EO, furthering the understanding of tissue-engineered endochondral bone formation.

\section{Materials and Methods}

\section{MSC culture}

MSCs were isolated from leftover material obtained from patients undergoing alveolar bone graft surgery (Medical Ethical Testing Commission approval code 2014-106; donor age range: 8-12 years old) following parental consent in accordance with relevant guidelines and regulations, as previously described (Knuth et al., 2018). Cells were plated at approximately 2,300 cells $/ \mathrm{cm}^{2}$ at $37{ }^{\circ} \mathrm{C}$ and $5 \% \mathrm{CO}_{2}$ in a humidified atmosphere in complete expansion medium [alpha modification minimum 
essential medium ( $\alpha$ MEM; Gibco) containing $10 \%$ foetal bovine serum (41Q204K; Gibco), $50 \mu \mathrm{g} / \mathrm{mL}$ gentamycin (Thermo Fisher Scientific), $1.5 \mu \mathrm{g} / \mathrm{mL}$ fungizone (Thermo Fisher Scientific), $25 \mu \mathrm{g} / \mathrm{mL}$ L-ascorbic acid 2-phosphate (Sigma-Aldrich) and $1 \mathrm{ng} / \mathrm{mL}$ fibroblast growth factor-2 (Instruchemie, Delfzijl, the Netherlands)]. Medium was replenished every 3-4 d until cells reached approximately $90 \%$ confluency. Then, they were passaged using $0.05 \%$ trypsin. Donor 1 and 2 were transdifferentiated following passage 6 and donor 3 was used at passage 7. At each passage, cells performed approximately 3 population doublings, with no difference among donors (data not shown). Population doublings were calculated by determining the $\log _{2}$ of the total cell number following growth for one passage, divided by the initial population count.

\section{Lentiviral generation}

MISSION ${ }^{\circledR}$ TRC1.5 shRNA targeting COL10A1 mRNA (TRCN0000082798; Sigma-Aldrich) and control plasmids [SHC001 (empty vector) and SHC002 (non-target control); Sigma-Aldrich] were transformed into DH5- $\alpha$ Escherichia coli (Invitrogen). shRNA targeting COL10A1 mRNA was analysed using the basic local alignment search tool on the NCBI website and no sequence homology with any other gene was identified. Likewise, no human homology was found for the non-target plasmid. Plasmids were isolated using an EndoFree Plasmid Maxi Kit (QIAGEN) as per manufacturer's instructions. HEK293FT cells were expanded in complete HEK medium [high-glucose Dulbecco's modified Eagle medium (DMEM; Gibco), $10 \%$ foetal bovine serum, $50 \mu \mathrm{g} / \mathrm{mL}$ gentamycin, $1.5 \mu \mathrm{g} / \mathrm{mL}$ fungizone] supplemented with $50 \mu \mathrm{g} / \mathrm{mL}$ geneticin (Thermo Fisher Scientific) and maintained at $37^{\circ} \mathrm{C}$ and $5 \% \mathrm{CO}_{2}$ in a humidified atmosphere. $24 \mathrm{~h}$ prior to transfection, $3 \times 10^{5}$ cells $/ \mathrm{cm}^{2}$ were plated in tissue culture Petri dishes (Corning) in high-glucose DMEM containing $10 \%$ foetal bovine serum. Cells were co-transfected with plasmids by calcium phosphate precipitation [3 $\mu$ g pLP1 plasmid, $3 \mu$ g pLP2 plasmid, $3 \mu \mathrm{g}$ pLP/VSVG plasmid (ViraPower Lentiviral Packaging Mix, Thermo Fisher Scientific) and 7.5 $\mu \mathrm{g}$ shRNA plasmid]. Medium was refreshed $24 \mathrm{~h}$ post transfection with HEK medium containing $20 \mathrm{mM}$ 4-(2-hydroxyethyl)-1-piperazineethanesulfonic acid (HEPES). Viral supernatant was harvested and filtered $(0.45 \mu \mathrm{m}$ filter $) 48 \mathrm{~h}$ following transfection and stored at $-80^{\circ} \mathrm{C}$ until use. p24 concentration was determined using INNOTEST ${ }^{\circledR}$ HIV Antigen mAb enzyme immunoassay (Fujirebio, Gent, Belgium) performed as per manufacture's instruction.

\section{Lentiviral transduction}

MSCs were transduced at $30 \%$ confluency. Cells were incubated for $48 \mathrm{~h}$ with 40 or $60 \mathrm{ng} / \mathrm{mL}$ p2 4 particles (depending on the batch used and the optimal dose determined by titration experiments) and $20 \mu \mathrm{g} / \mathrm{mL}$ protamine sulphate (Sigma-Aldrich) in complete expansion medium (Fig. 1-3). Following incubation, cells were washed, expanded and selected after reaching $80 \%$ confluency. Selection was achieved in $72 \mathrm{~h}$ using $5 \mu \mathrm{g} / \mathrm{mL}$ puromycin in complete expansion medium. After selection, cells were washed and expanded until $90 \%$ confluency, when they were either harvested for RNA [homogenised in $350 \mu \mathrm{L}$ Trizol (Thermo Fisher Scientific) and stored at $-80{ }^{\circ} \mathrm{C}$ until isolated] or used for differentiation studies. p24 particle, protamine sulphate and puromycin concentrations were determined prior to experimental start through titration experiments (Fig. 1-3). Knock-down percentage was calculated by subtracting the average COL10A1 expression in COL10A1 knock-down pellets from the average COLX expression in non-treated pellets. This was divided by the average of the non-treated COL10A1 expression and then multiplied by 100 to obtain a percentage.

\section{Chondrogenic differentiation}

MSCs were chondrogenically differentiated in complete chondrogenic medium [high-glucose DMEM supplemented with $50 \mu \mathrm{g} / \mathrm{mL}$ gentamycin, $1.5 \mu \mathrm{g} / \mathrm{mL}$ fungizone, $1 \mathrm{mM}$ sodium pyruvate (Gibco), $40 \mu \mathrm{g} / \mathrm{mL}$ proline (Sigma-Aldrich), $1: 100 \mathrm{v} / \mathrm{v}$ insulintransferrin-selenium (ITS+; BD Biosciences), 10 ng/ $\mathrm{mL}$ transforming growth factor $\beta 3$ (Peprotech), $25 \mu \mathrm{g} / \mathrm{mL}$ L-ascorbic acid 2-phosphate and $100 \mathrm{nM}$ dexamethasone (Sigma-Aldrich)] using $2 \times 10^{5} \mathrm{MSCs}$ resuspended in $15 \mathrm{~mL}$ polypropylene tubes and centrifuged at $300 \times g$ for $8 \mathrm{~min}$. Cultures were maintained in a humidified atmosphere at $37{ }^{\circ} \mathrm{C}$ and $5 \% \mathrm{CO}_{2}$ and medium was replenished every 3-4 d for $21 \mathrm{~d}$. Samples were harvested following 7 and $21 \mathrm{~d}$ in culture for RNA isolation (as previously described), glycosaminoglycan (GAG)/DNA analysis (stored at $-80{ }^{\circ} \mathrm{C}$ ), Western blot (snap-frozen), histology ( $4 \%$ formalin fixed for $2 \mathrm{~h}$ ), enzyme-linked immunosorbent assay (ELISA) (supernatant) or migration (conditioned medium; described below).

\section{Osteogenic differentiation}

MSCs were plated at a density of $3 \times 10^{3}$ cells $/ \mathrm{cm}^{2}$ in expansion medium. $24 \mathrm{~h}$ after plating, the medium was changed to osteogenic induction medium (high-glucose DMEM supplemented with $10 \%$ $\mathrm{v} / \mathrm{v}$ foetal bovine serum, $50 \mu \mathrm{g} / \mathrm{mL}$ gentamycin, $1.5 \mu \mathrm{g} / \mathrm{mL}$ fungizone, $10 \mathrm{mM}$ glycerol-3-phosphate, $0.1 \mu \mathrm{M}$ dexamethasone and $0.1 \mathrm{mM}$ L-ascorbic acid 2-phosphate). Cultures were maintained at $37^{\circ} \mathrm{C}$ and $5 \% \mathrm{CO}_{2}$ in a humidified atmosphere. Medium was refreshed every 3-4 d for 13-21 d. Once cell sheets began to detach from the well outer periphery, culture was ended and samples were fixed in $4 \%$ $(\mathrm{w} / \mathrm{v})$ formalin. von Kossa staining was performed as previously described (Knuth et al., 2018).

\section{Adipogenic differentiation}

MSCs were plated at a density of $2.1 \times 10^{4}$ cells/ $\mathrm{cm}^{2}$ in adipogenic induction medium [high-glucose 
Table 1. Primer sequences used for real time PCR. B2M: beta-2-microglobulin; VEGFa: vascular endothelial growth factor a; COL1A1: collagen type I; COL2A1: collagen type II; COL10A1: collagen type X; BMP2: bone morphogenetic protein 2; MMP13: matrix metallopeptidase 13; AGCN: aggrecan.

\begin{tabular}{|c|c|c|}
\hline Gene names & Forward sequence $\left(5^{\prime}-3^{\prime}\right)$ & Reverse sequence $\left(5^{\prime}-3^{\prime}\right)$ \\
\hline$B 2 M$ & TGCTCGCGCTACTCTCTCTTT & TCTGCTGGATGACGTGAGTAAAC \\
\hline$V E G F a$ & CTTGCCTTGCTGCTCTACC & CACACAGGATGGCTTGAAG \\
\hline COLIA1 & CAGCCGCTTCACCTACAGC & TTTTGTATTCAATCACTGTCTTGCC \\
\hline COL2A1 & CCСCATCTGCCCAACTGA & СTCCTTTCTGTCCCTTTGGT \\
\hline COL10A1 & АСТTСТСТТАССАСАТАCACG & CCAGGTAGCCCTTGATGATGTACT \\
\hline BMP2 & AACACTGTGCGCAGCTTCC & CTCCGGGTTGTTTTCCCAC \\
\hline MMP13 & AAGGAGCATGGCGACTTCT & TGGCCCAGGAGGAAAAGC \\
\hline$A G C N$ & TCGAGGACAGCGAGGCC & TCGAGGGTGTAGCGTGTAGAGA \\
\hline
\end{tabular}

DMEM supplemented with $10 \%$ foetal bovine serum, $50 \mu \mathrm{g} / \mathrm{mL}$ gentamycin, $1.5 \mu \mathrm{g} / \mathrm{mL}$ fungizone, $0.2 \mathrm{mM}$ indomethacin (Sigma-Aldrich), $0.01 \mathrm{mg} / \mathrm{mL}$ insulin (Sigma-Aldrich), $0.5 \mathrm{mM} 3$ iso-butyl-1-methylxanthine (Sigma-Aldrich) and $1 \mu \mathrm{M}$ dexamethasone]. Cultures were maintained at $37^{\circ} \mathrm{C}$ and $5 \% \mathrm{CO}_{2}$ in a humidified atmosphere. Medium was refreshed as every 3-4 d for 19-21 d. Then, samples were prepared for histology [4\% (w/v) formalin-fixed]. Oil red O staining was performed as previously described (Knuth et al., 2018).

\section{Cell viability testing}

MSCs were plated at a density of $2.1 \times 10^{4} \mathrm{cells} / \mathrm{cm}^{2}$ and treated for $48 \mathrm{~h}$ at $37^{\circ} \mathrm{C}, 5 \% \mathrm{CO}_{2}$ in a humidified atmosphere with either protamine sulphate $(10$, $25,50,75$ or $100 \mu \mathrm{g} / \mathrm{mL}$ ) or puromycin $(1.25,2.5$, $5,7.5$ or $10 \mu \mathrm{g} / \mathrm{mL}$ ) in MSC expansion medium. Then, cells were washed with phosphate-buffered saline (PBS) and incubated in the dark for $3 \mathrm{~h}$ at $37{ }^{\circ} \mathrm{C}$ and $5 \% \mathrm{CO}_{2}$ in a humidified atmosphere in $0.5 \mathrm{mg} / \mathrm{mL}$ MTT (3-[4,5-dimethylthiazol-2-yl]-2,5diphenyl tetrazolium bromide; Sigma-Aldrich)supplemented MSC expansion medium. Next, medium was aspirated and cells were washed with PBS. MTT-tetrazolium crystals formed by healthy cells were dissolved in $100 \%$ ethanol. Absorbance was measured at $570 \mathrm{~nm}$ and $670 \mathrm{~nm} ; 570: 670$ ratio was compared to non-treated control MSCs.

\section{Western blot}

Pellets were snap-frozen in liquid nitrogen and homogenised in $100 \mu \mathrm{L}$ M-PER (Mammalian Protein Extraction Reagent, Thermo Fisher Scientific) with 1× Halt $^{\mathrm{TM}}$ Protease Inhibitor Cocktail (Thermo Fisher Scientific) followed by water bath sonication (10 cycles of 30 s sonication and $30 \mathrm{~s}$ rest). Total protein was measured using the Pierce ${ }^{\mathrm{TM}}$ BCA Protein Assay Kit (Thermo Fisher Scientific). $10 \mu \mathrm{g}$ total protein was incubated at $95{ }^{\circ} \mathrm{C}$ and separated on a gradient gel (Bolt 4-12 \% Bis-Tris Plus, Thermo Fisher Scientific) under non-reducing conditions. Proteins were transferred to a polyvinylidene fluoride or polyvinylidene difluoride (PVDF) membrane and blocked with $5 \% \mathrm{w} / \mathrm{v}$ fat-free milk in $0.1 \% \mathrm{v} / \mathrm{v}$ tris-buffered saline (TBS)-Tween 20. The membrane was cut at $70 \mathrm{kDa}$ and each piece was incubated overnight with its respective antibody: 1 : 1,000 mouse monoclonal anti-COLX, clone X53 (Quartett, Berlin, Germany) and 1 : 2,000 rabbit monoclonal anti $\alpha$-tubulin (Cell Signaling). Next, incubation for $1 \mathrm{~h}$ at room temperature with the respective secondary antibody was performed: $1:$ 2,000 horseradish peroxidase (HRP)-conjugated goat anti-mouse and $1: 2,000 \mathrm{HRP}$-conjugated goat anti-rabbit (both Thermo Fisher Scientific). Protein bands were visualised using the Super Signal West detection kit (Thermo Fisher Scientific) on the digital $\Delta$ camera Alliance 2.7 (Uvitec, Cambridge, UK) with an exposure time of 15 (COLX) or $17 \mathrm{~min}$ ( $\mu$-tubulin).

\section{RNA isolation and gene expression}

Samples were homogenised in $350 \mu \mathrm{L}$ Trizol to which $70 \mu \mathrm{L} 100 \%$ chloroform was added and thoroughly mixed. Following a $10 \mathrm{~min}$ incubation at room temperature and phase separation at $10,000 \times g$ for $15 \mathrm{~min}$, the aqueous phase was mixed with an equal volume of $70 \%$ ethanol and transferred onto an RNeasy ${ }^{\circledR}$ kit column. RNA was isolation and purified using the RNeasy ${ }^{\circledR}$ Micro Kit (QIAGEN) following manufacturer's instructions. cDNA was reversetranscribed as per manufacturer's instruction using a First Strand cDNA Synthesis Kit (Thermo Fisher Scientific) and PCR was performed as previously described (Table 1) (Knuth et al., 2015. Mesenchymal stem cell-mediated endochondral ossification utilising micropellets and brief chondrogenic priming, towards scale-up. Proceedings of the 24th NBTE Annual Meeting, Lunteren, The Netherlands. pp: 65). For gene expression of monolayer, day 7 and day 14 of chondrogenic differentiation, 3 different MSC donors were analysed. For gene expression at day 21, only one MSC donor was analysed (3 pellets/ donor/condition). Gene expression is shown as $\Delta \Delta \mathrm{CT}$.

\section{Biochemical assay}

Chondrogenically differentiated samples stored at $-80^{\circ} \mathrm{C}$ were digested overnight at $60{ }^{\circ} \mathrm{C}$ in buffer containing papain $\left[0.2 \mathrm{M} \mathrm{NaH} \mathrm{PO}_{4^{\prime}}\right.$ $0.01 \mathrm{M}$ ethylenediaminetetraacetic acid (EDTA), 
$0.01 \mathrm{M}$ cysteine $\mathrm{HCl}, 250 \mu \mathrm{g} / \mathrm{mL}$ papain (SigmaAldrich), $\mathrm{pH}$ 6]. Then, GAGs were quantified by dimethylmethylene blue assay ( $\mathrm{pH} 3$ ) with chondroitin sulphate C (Sigma-Aldrich) as a standard, utilising an absorbance 530 : 590 ratio. Ethidium bromide was used to determine sample DNA content. Calf thymus DNA (Sigma-Aldrich) was used as a standard.

\section{Cytokine quantification}

Culture medium was refreshed $24 \mathrm{~h}$ before harvesting the pellets. Supernatant was applied to a human cytokine angiogenic multiplex chemiluminescent ELISA (150251HU; Quansys Biosciences, Logan, UT, USA) as per manufacturer's instructions. This angiogenic multiplex included the following targets: angiotensin II (Ang-2), fibroblast growth factor-2 (FGF-2), hepatocyte growth factor (HGF), interleukin-8 (IL-8), platelet derived growth factorBB (PDGF-BB), tissue inhibitor of metalloproteinase 1 and 2 (TIMP-1 and TIMP-2), tumour necrosis factor (TNF). The ELISA plate was imaged using the Amersham Imager 600 (GE Healthcare) and images were quantified using the $\mathrm{Q}$-view software ${ }^{\circledR}$ provided by the ELISA manufacturer (Quansys Bioscience).

\section{Histology}

In vitro samples were fixed for $2 \mathrm{~h}$ in $4 \%$ formalin prior to paraffin-wax embedding. In vivo samples were formalin-fixed for $24 \mathrm{~h}$ and decalcified for $10 \mathrm{~d}$ in $10 \%$ EDTA w/v in $\mathrm{H}_{2} \mathrm{O}$ refreshed every other day. Following paraffin-wax embedding, $6 \mu \mathrm{m}$-thick sections were cut from all samples. Sections were deparaffinised and stained with haematoxylin-eosin ( $\mathrm{H} \& \mathrm{E})$, safranin $\mathrm{O}$, tartrateresistant acid phosphatase (TRAP) and antibodies against COLII or COLX, as previously described (Knuth et al., 2015. Mesenchymal stem cell-mediated endochondral ossification utilising micropellets and brief chondrogenic priming, towards scale-up. Proceedings of the 24th NBTE Annual Meeting, Lunteren, The Netherlands. pp: 65).

\section{Subcutaneous implantation}

Experiments were conducted with approval by the Animal Experiments Committee at the Erasmus Medical Centre, Rotterdam (EU license number 15-114-02) adhering to all relevant guidelines and regulations. 8 week-old athymic nude mice (Balb/c nudes, Charles River) were housed under standard light-dark cycles with food and water ad libitum (12 mice in total; housed in groups of 2). Pain medication (buprenorphine $0.05 \mathrm{mg} / \mathrm{kg}$ body weight) was administered pre-operatively under anaesthesia (1-3\% isoflurane). Dorsal incisions were created in which a subcutaneous pocket was made. 4 subcutaneous pockets were created in the back of each mouse through two incisions, one in the upper and one in lower back. At each incision, 2 pockets (left and right) were created by blunt dissection. 3 control or virally transduced pellets per pocket were implanted subcutaneously (conditions were randomised among mice and physical positions). 3 implantations were performed per experimental condition ( 3 pellets or 600,000 cells/implantation). Incisions were closed using staples. Animals were euthanised 8 or 10 weeks post implantation by $\mathrm{CO}_{2}$ asphyxiation.

\section{X-ray micro-computed tomography ( $\mu \mathrm{CT})$ longitudinal imaging}

$\mu \mathrm{CT}$ scans were performed at the Applied Molecular Imaging Erasmus MC facility using the Quantum FX (Perkin-Elmer). While animals were anaesthetised (1$2.5 \%$ isoflurane), scans were acquired using a field of view of $60 \mathrm{~mm}(90 \mathrm{kV} / 160 \mathrm{~mA}, 4.5 \mathrm{~min})$ bi-weekly. Using the Analyze 11.0 software (AnalyzeDirect, Nieuwe Niedorp, the Netherlands), scans were quantified by converting original linear attenuation coefficient measurements by linear transformation to Hounsfield units (HU). Median spatial filters were applied with a $3 \times 3 \times 3$ kernel size. Values corresponding to a density of over $0.133 \mathrm{~g} / \mathrm{cm}^{3}$ (determined by phantom scans) were segmented out and quantified as calcified tissue.

\section{Figures and statistical analysis}

Graph figures were created using GraphPad Software (La Jolla, CA, USA). Graphs depict $\triangle$ CT values (Livak and Schmittgen, 2001), setting non-treated value to 1. Linear mixed model with Bonferroni post-hoc comparison was performed using SPSS (Ver 21. SPSS Inc) on expansion data in Fig. 1.

\section{Results}

Lentivirally transduced MSCs maintain proliferative and differentiation capacity following puromycin selection

Concentrations of protamine sulphate $(20 \mathrm{ng} / \mathrm{mL})$ and puromycin $(5 \mu \mathrm{g} / \mathrm{mL})$ were optimised by titration experiments (data not shown). Protamine sulphate was used to aid trafficking of the lentivirus particles into the cell by neutralising the charge between the MSC membrane and viral envelope. Puromycin was used to select only transduced cells due to the presence of a puromycin resistance cassette in the lentivirus. Cells not containing the cassette, meaning non-transduced cells, were killed in the presence of puromycin. Minimal doses of both drugs were chosen to avoid unnecessary cell stress during transduction. For each viral batch, the optimal concentration (as determined by p24 viral antigen concentration) was determined based on minimum dose at which cells maintained proliferative capacity (compared to nontransduced controls from the same donor) following selection. Following transduction with optimal lentiviral and protamine sulphate concentrations, MSCs maintained a healthy spindle-like morphology, which was retained after puromycin selection (data not shown). MSCs recovered quickly from selection 
stress and all grew at a similar rate, undergoing comparable population doublings as non-treated controls (Fig. 1a,b). Furthermore, transduced cells maintained their differentiation potential upon osteogenic and adipogenic induction (Fig. 1c). Chondrogenesis as well as hypertrophy was observed by day 7 as evidenced by a clear upregulation in COL2A1, COL10A1 and COL1A1 expression (Fig. 1d). The expression of COL2A1 and COL10A1 was strongly affected by COL10A1 knock-down (Fig. 1d).

\section{COL10A1 could be efficiently knocked-down by lentivirally delivered shRNA}

MSCs from each condition were chondrogenically differentiated for $21 \mathrm{~d}$ by pellet culture. Following differentiation, COL10A1 expression was effectively knocked-down with an efficiency ranging between 80 and $99 \%$, depending on the MSC donor (Fig. 2a; donor 1: > $99 \%$; donor 2: $99 \%$; donor 3: $80 \%$ ). Gene and protein expressions of COLX were significantly downregulated as compared to non-

a

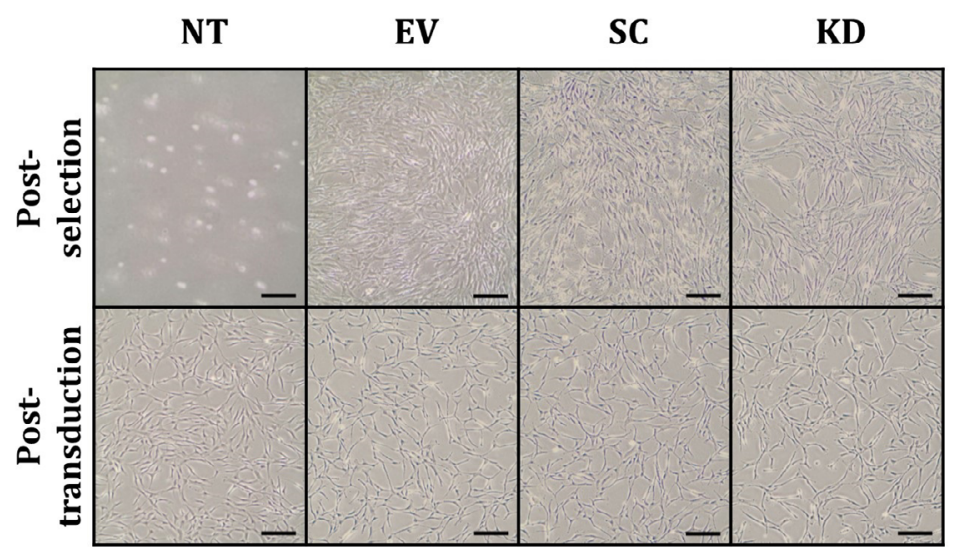

b

C

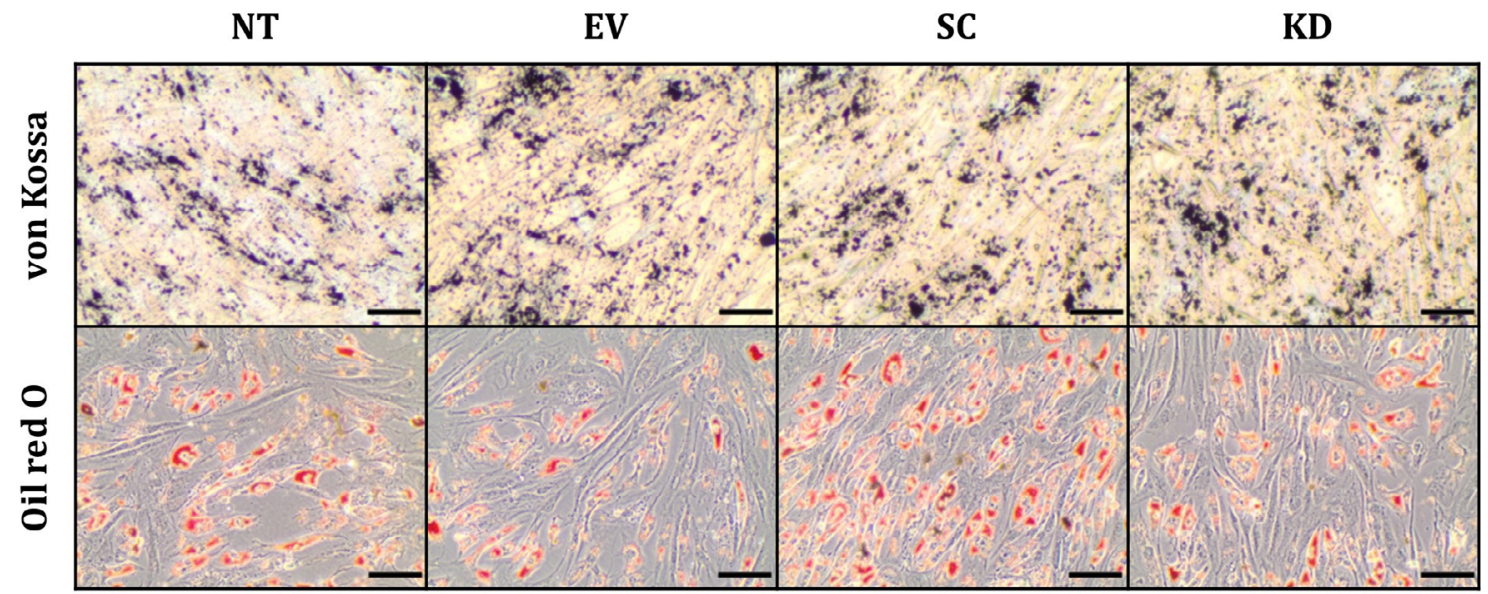

d
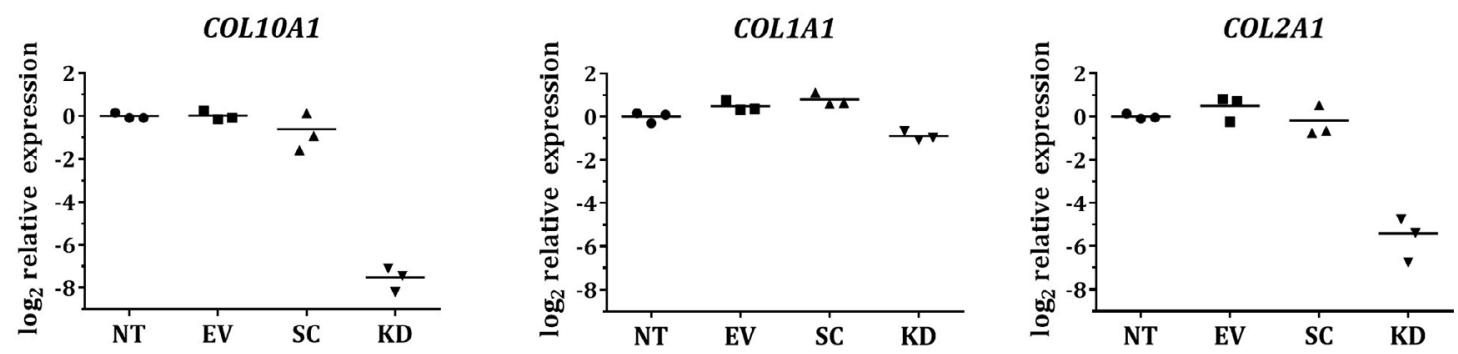

Fig. 1. Lentiviral infection did not hinder MSC expansion or differentiation capabilities. (a) MSCS were transduced with lentivirus containing an empty vector, non-target shRNA or COL10A1 shRNA. Then, MSCs were selected based on puromycin resistance. Following transduction, cells maintained MSC-like morphology. Post-selection, positively infected cells maintained both morphology and proliferative capacity; scale bar: $200 \mu \mathrm{m}$. (b) Transduced MSCs post-selection expanded at a similar rate to non-treated MSCs, undergoing a similar number of population doublings $(n=4$; linear mixed model with Bonferroni post-hoc comparison: non-significant). (c) Transduced MSCs maintained the capacity to differentiate osteogenically and adipogenically. Scale bar: $50 \mu \mathrm{m}$. (d) mRNA expression of COL10A1, COL1A1 and COL2A1 following $7 \mathrm{~d}$ of chondrogenic differentiation in donor 1. NT: non-treated; EV: empty vector; SC: non-target control; KD: knock-down. 
treated, empty vector and non-target controls, which exhibited normal expression levels for all donors (Fig. 2a-c). COLX downregulation was confirmed by immunohistochemistry and Western blot for all donors and, therefore, both virus batches used (Fig. 2b,c). COL10A1 knock-down pellets from donor 1 were too small to be processed for immunohistochemistry (Fig. 2c; donor 1). A complete absence of positive staining in COL10A1 knock-down pellets from donor 2 and a slight positivity in the pellets from donor 3 were observed; however, the quantity was far less than that observed in controls (Fig. 2c; donor 2, donor 3). Overall, the validity of the silencing approach was demonstrated at both transcript and protein level.

\section{The absence of COL10A1 during chondrogenic} differentiation of MSCs affected the ECM

After confirming the efficiency of COL10A1 knockdown, how chondrogenic differentiation of MSCs was affected in its absence was investigated. For all donors, non-treated, empty vector and non-target

a
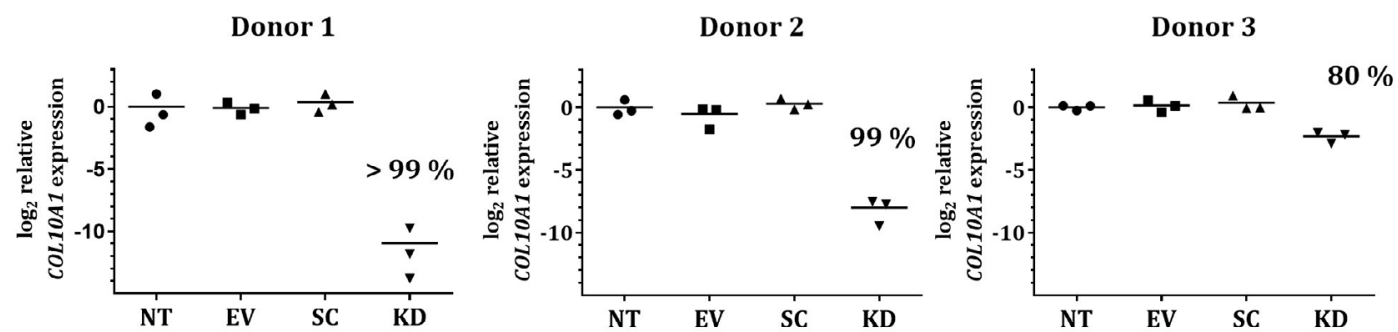

b
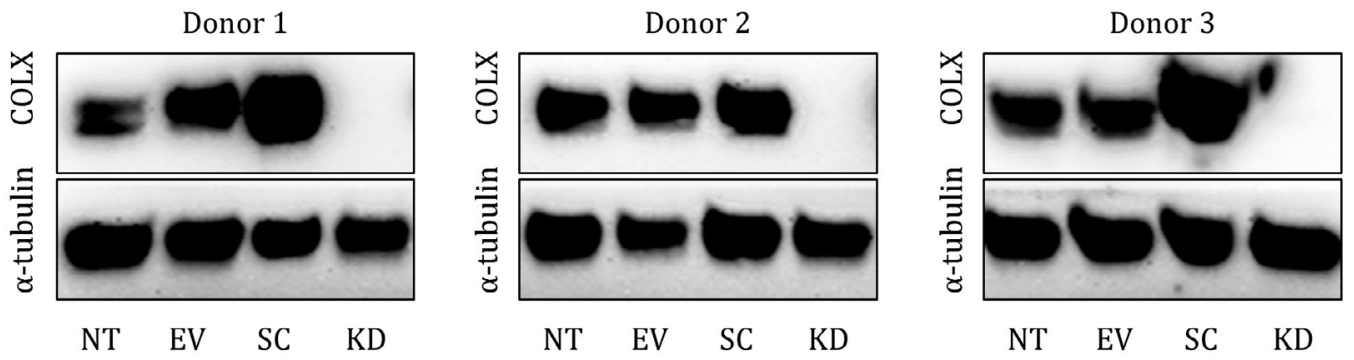

C

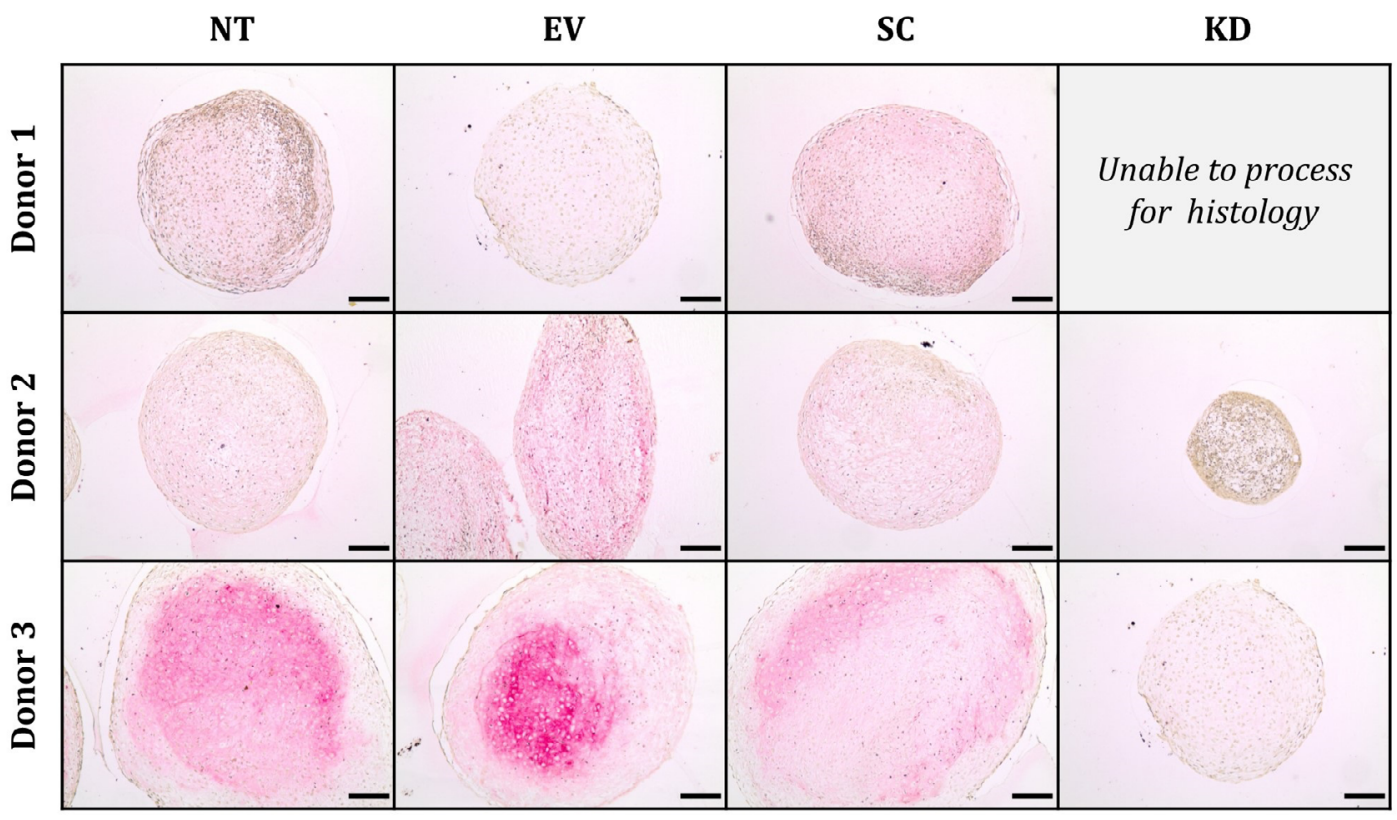

Fig. 2. COL10A1 can be efficiently knocked-down using lentivirally delivered shRNA. MSCs were transduced with lentivirus containing an empty vector, non-target shRNA or COL10A1 shRNA. Then, MSCs were selected based on puromycin resistance, expanded and chondrogenically differentiated for $21 \mathrm{~d}$, when they were harvested for analysis. (a) Compared to control conditions, MSCs transduced with shRNA directed against COL10A1 showed significant down regulation of gene expression, with the level of knock-down varying between donors (\% NT vs. COLX KD; pooled data: linear mixed model with Bonferonni correction: a $p$ 0.05). (b) This down regulation was also observed at the protein level by Western blot and (c) COLX immunohistochemistry. Scale bar: $200 \mu \mathrm{m}$. NT: non-treated; EV: empty vector; SC: non-target control; KD: knock-down. 
controls maintained their chondrogenic potential, indicating that lentiviral transduction and RNAinduced silencing complex activation did not negatively affect chondrogenic differentiation of MSCs (Fig. 3). COL10A1 knock-down pellets were significantly smaller than all controls for all donors, prompting the investigation of whether the absence of COLX affected other matrix components. When COLX production was mostly absent, as in donors 1 and 2, a significant decrease in GAGs (Fig. 3b) and $A C A N$ expression (Fig. 3c) was observed. However, when a less effective knock-down of COL10A1 was achieved, as in donor 3 , matrix production was less affected (Fig. 3). A similar trend was observed with regard to COLII at both protein (Fig. 4a) and gene expression (Fig. 4b) level. A similar result was observed for COL1A1 (Fig. 4c). Overall, COL10A1 knock-down affected the production of crucial chondrogenic ECM components following differentiation.

\section{A complete cartilage template was essential for tissue-engineered MSC-mediated EO}

After observing that COL10A1 knock-down in MSCs affected tissue-engineered cartilage formation and confirming that control conditions for each

a

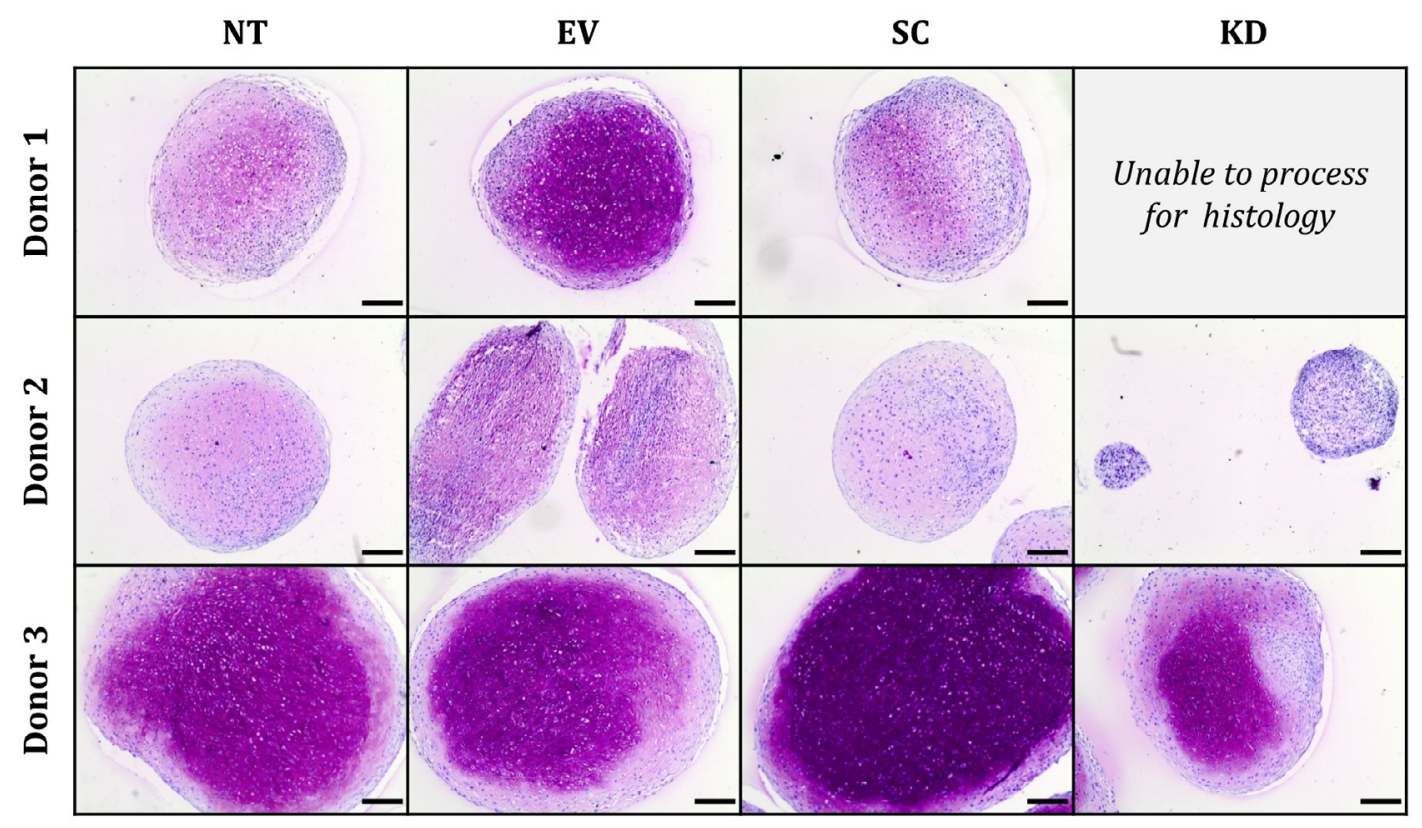

b
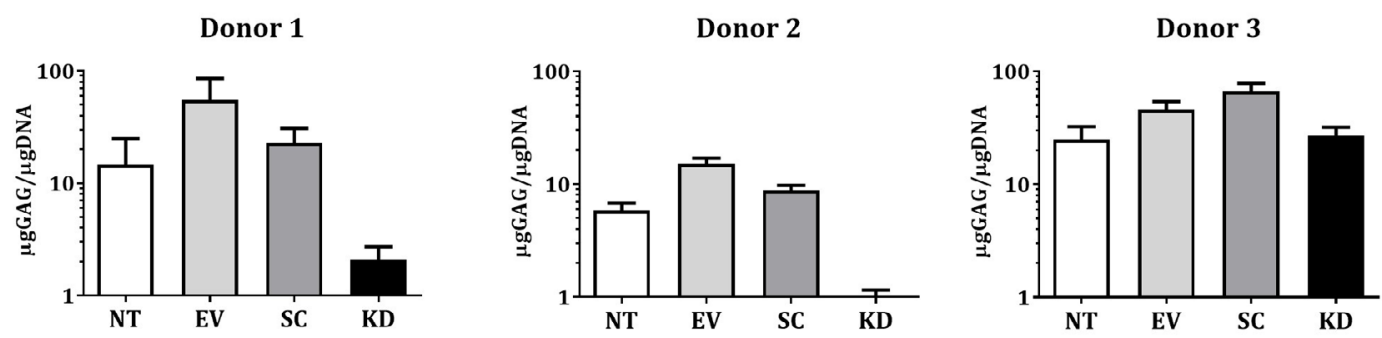

C
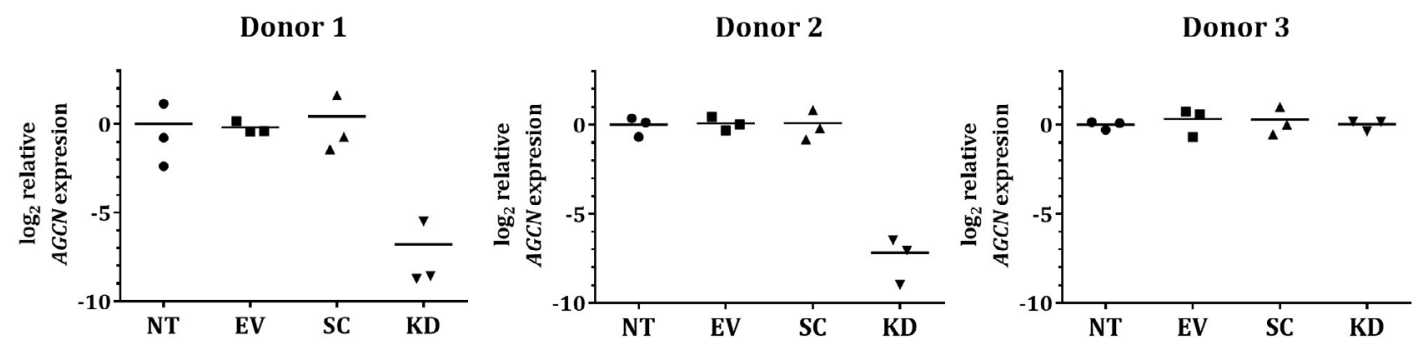

Fig. 3. A small quantity of COL10A1 was required for the production of GAGs during chondrogenic differentiation of MSCs. (a) Thionine staining and GAG quantification showed that, upon over $99 \%$ COL10A1 knock-down, GAG production was decreased (donors 1 and 2). (b) Production of $20 \%$ of the original COL10A1 quantity was enough to trigger GAG production (donor 3). (c) A similar pattern was also observed for $A C A N$ expression. Staining and expression of genes did not vary greatly among non-treated, empty and non-target conditions. Scale bar: 200 Mm. NT: non-treated; EV: empty vector; SC: non-target control; KD: knock-down. 
donor were unaffected, how a lack of COL10A1 affected endochondral bone formation following subcutaneous implantation in nude mice, a model shown to result in endochondral bone formation (Farrell et al., 2011; Knuth et al., 2017), was determined. Chondrogenically differentiated MSC pellets were cultured for $21 \mathrm{~d}$ in vitro and implanted subcutaneously in nude mice. All control conditions from these donors underwent mineralisation (Fig. 5). COL10A1 knock-down pellets from donors 1 and 2 did not show in vivo mineralisation in $\mu$ CT scans (Fig. 5b). No pellets containing COL10A1 shRNA were retrieved from donor 1 and only 1 of the 9 implanted pellets was retrieved from donor 2 . This pellet showed calcification around the outer periphery but no marrow formation and was substantially smaller than control pellets (Fig 5a, donor 2). All pellets from donor 3 containing shRNA against COL10A1 not only provided evidence of calcification in $\mu \mathrm{CT}$ scans (Fig. 5 b; donor 3$)$, albeit less than that observed in other conditions, but also after 10 weeks in vivo showed the presence of mature bone, bone marrow and calcified cartilage.

An almost complete down-regulation of COL10A1 in MSCs pre-implantation hindered in vivo bone formation; however, when down-regulation was not as efficient, bone formation proceed normally.

a
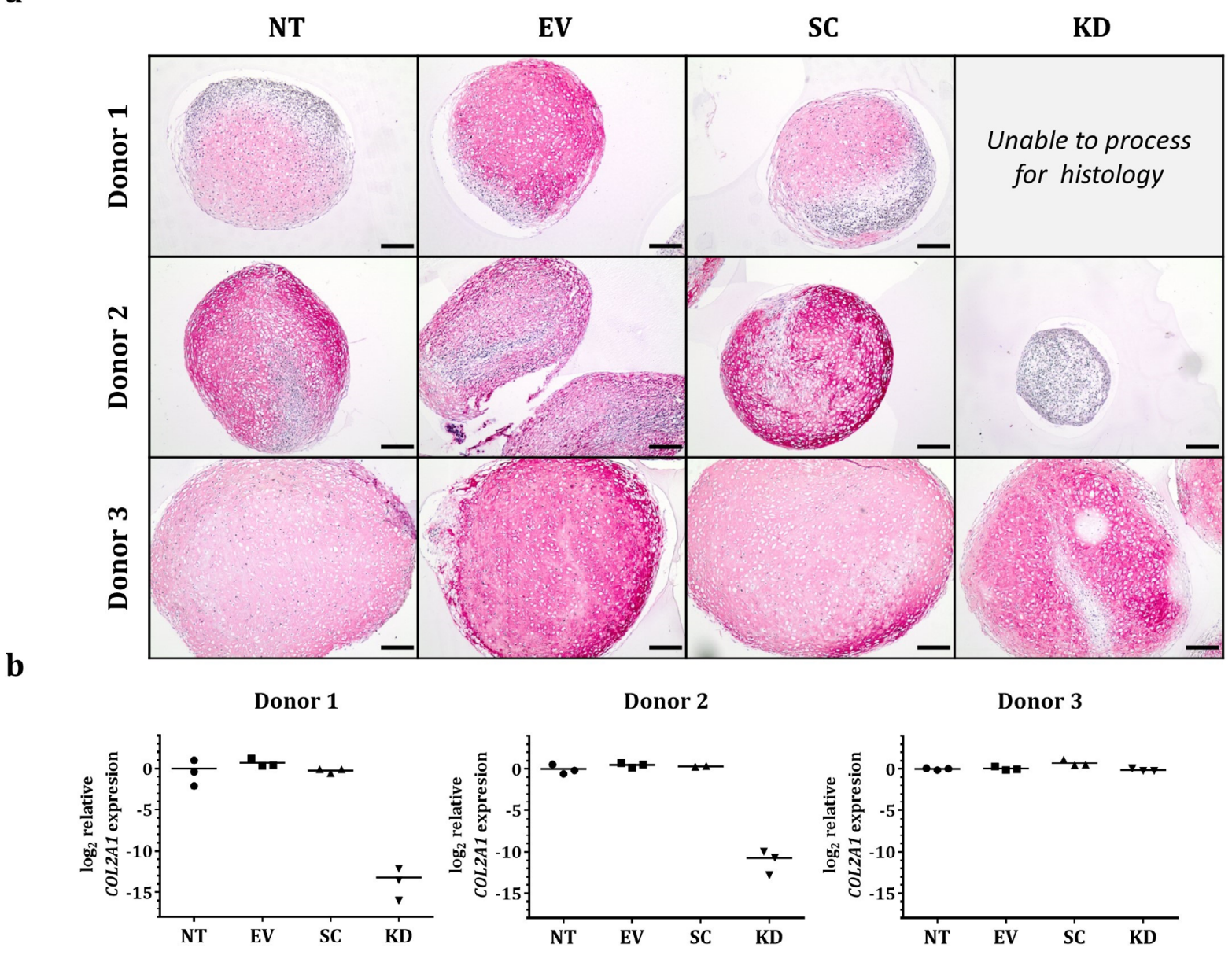

C
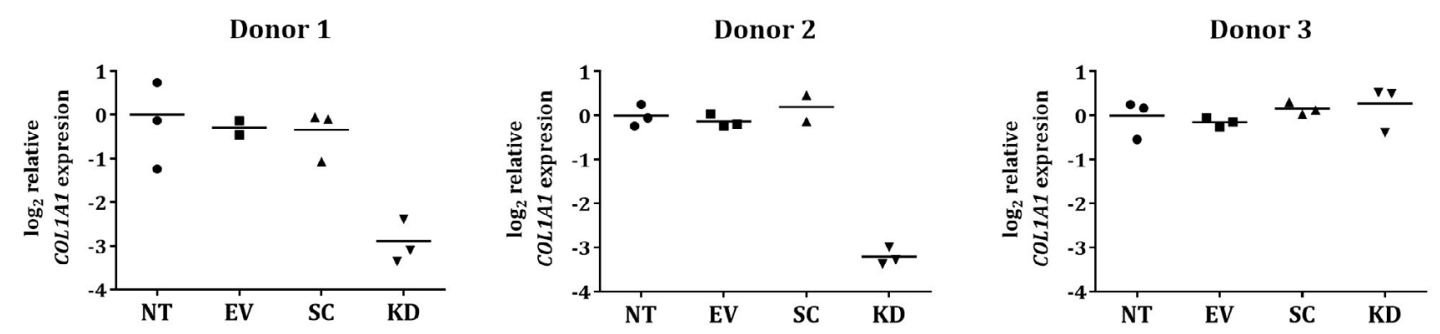

Fig. 4. A small quantity of COL10A1 was required for proper COLII production during chondrogenic differentiation of MSCs. (a) COLII immunohistochemistry and (b) gene expression showed that, when COL10A1 was knocked-down over $99 \%$, COL2A1 production was affected (donors 1 and 2). When the knockdown was less efficient (donor 3), COL2A1 production was not affected. (c) The same trend was observed for COL1A1. Staining and expression of genes did not vary greatly between non-treated, empty and non-target conditions. Scale bar: 200 m. NT: non-treated; EV: empty vector; SC: non-target control; KD: knock-down. 
Chondrogenic matrices with decreased COL10A1 exhibited decreased osteoclastic remodelling

As in vivo endochondral bone formation was differentially affected depending on the efficiency of COL10A1 knock-down, next step was to determine if the presence of osteoclasts - important for matrix remodelling, a central process to successful endochondral bone formation (Ortega et al., 2004) - was also affected in a similar manner. TRAP was performed and a different staining pattern was observed in donor 2 COL10A1-shRNA-containing pellet versus controls (Fig. 6). Non-treated, empty vector and scrambled pellets showed positive staining in the central marrow cavity whereas COL10A1shRNA-containing pellets only showed staining around the outer periphery. This was starkly different from COL10A1-shRNA-containing pellets from donor 3 which exhibited a similar staining pattern to what was observed in all different control condition pellets (Fig. 6). When COL10A1 expression was significantly knocked-down, less osteoclasts were observed in vivo; however, this could be an indirect rather than direct effect of COL10A1 knock-down. Further research will be required to determine this.

\section{Down-regulation of COL10A1 affected the secretion profile of chondrogenic MSCs}

Following implantation, some calcification and altered cell recruitment were observed in COL10A1 knockdown pellet from donor 2 . Less extensive differences were observed in COL10A1 knock-down pellets from donor 3 (Fig. 7). In order to further understand why these differences occurred, the secreted profile of factors produced by chondrogenic MSCs and influencing EO was investigated. PDGF-BB and FGF2, two factors involved in endothelial migration and

a

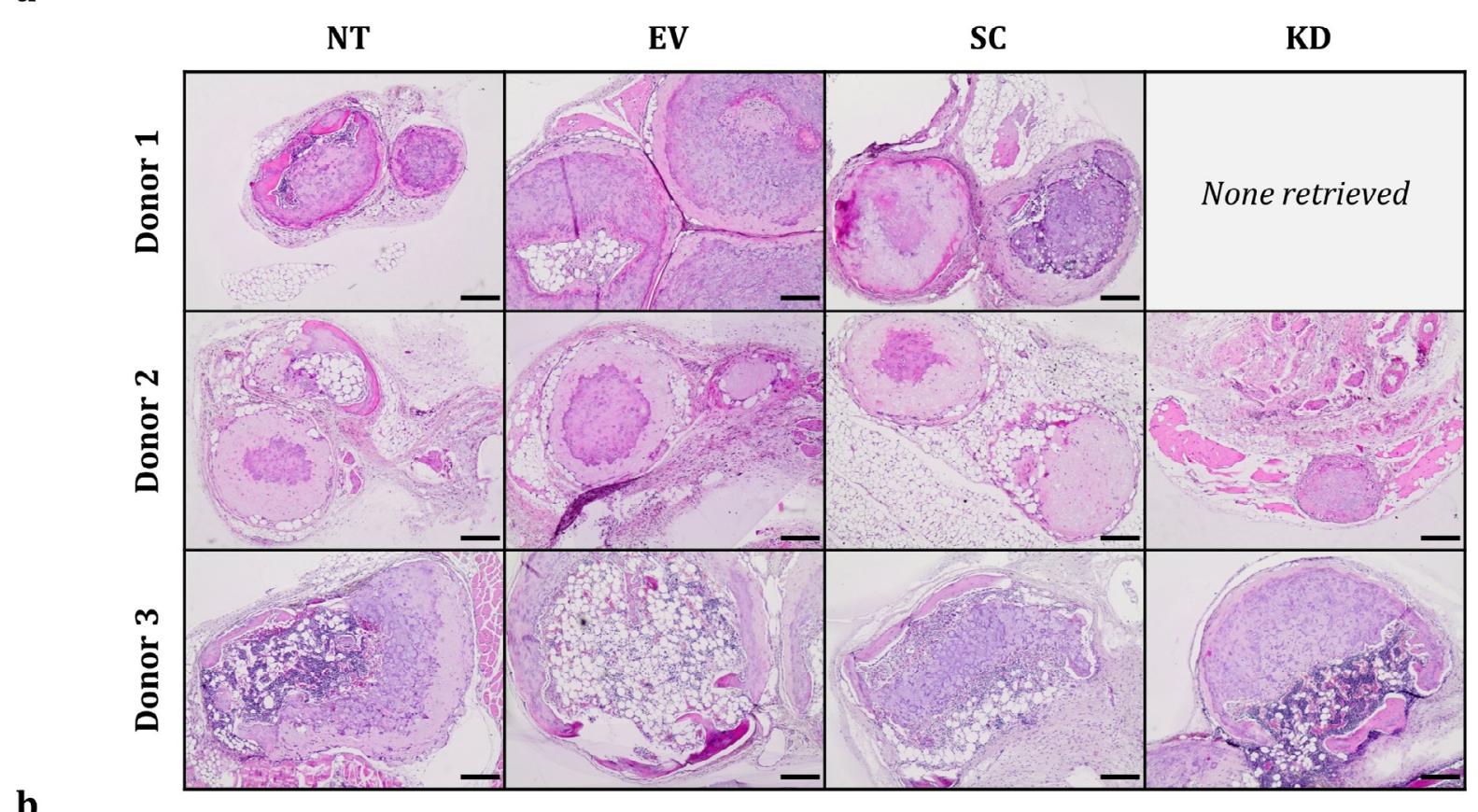

b
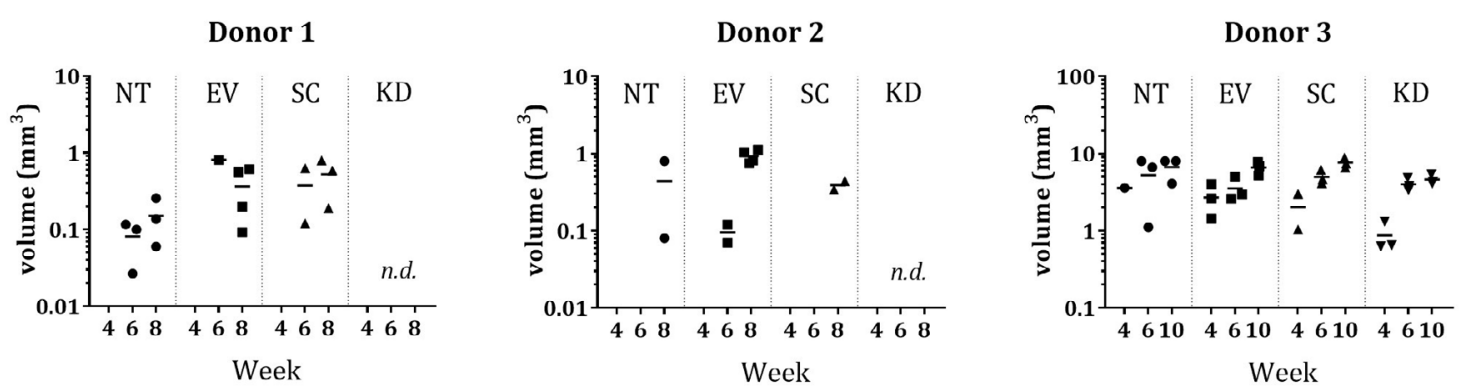

Fig. 5. When COL10A1 production was absent in chondrogenically differentiated MSCs, bone formation was hindered. However, a less significant knock-down resulted in normal bone formation. (a,b) Following 8 (donors 1 and 2) or 10 weeks (donor 3 ) of in vivo implantation, control conditions showed the production of bone, bone marrow and calcified cartilage. Donor 1 COL10A1 knock-down pellets could not be retrieved following implantation; however, one pellet from donor 2 showed signs of mineralisation. Pellets from donor 3 appeared to be similar to control conditions; however, mineralisation was initially delayed, as observed in longitudinal $\mu \mathrm{CT}$. Given that the samples were retrieved at 8 weeks for donors 1 and 2 and at 10 weeks for donor 3, it was not possible to pool the donors for statistical analysis. Scale bar: $200 \mu \mathrm{m}$. NT: non-treated; EV: empty vector; SC: non-target control; KD: knock-down; n.d.: non-detectable. 
osteoblastic migration/maturation (Kim et al., 2007; Ornitz, 2005; Xie et al., 2014), were lowly expressed (Fig. 7a,b). These factors were both detected at a higher level in donors 2 and 3. A similar trend was observed for TNF $\alpha$, a factor linked to inflammation and osteoclastic maturation (Gerstenfeld et al., 2003; Kimble et al., 1997) (Fig. 7c). This showed that the secreted profile of chondrogenically differentiated MSCs, at least in part, was influenced by COL10A1 production.

\section{Discussion}

The role of COLX in EO is often debated. Where some developmental studies have found significant perinatal death, delayed bone formation and a compromised immune system, others have reported no major difference between knock-out and wildtype controls (Jacenko et al., 2001; Jacenko et al., 2002; Kwan et al., 1997; Rosati et al., 1994). In these studies, matrix production was significantly altered, resulting in the distribution of matrix components around hypertrophic cells and throughout the growth plate (Jacenko et al., 2001; Jacenko et al., 2002). COLX also plays a role in the movement and accumulation of proteoglycans and associates with other collagens within the matrix (Chen et al., 1990; Chen et al., 1992; Poole and Pidoux, 1989). However, how COLX contributes to tissue-engineered cartilage formation and subsequent endochondral bone formation has yet to be determined. The present study investigated the role of COLX from a tissue engineering perspective using culture-expanded MSCs. Using chondrogenically differentiated MSCs, which are prone to hypertrophic differentiation (Mueller and Tuan, 2008; Narcisi et al., 2015; Pelttari et al., 2006), GAG quantity was found to be significantly decreased and other major ECM components, including COLII and aggrecan, significantly downregulated or almost absent when COLX knock-down was more than $80 \%$ (gene expression). As chondrogenically differentiated MSCs express COLX early during differentiation and have previously been shown to form bone following in vivo implantation (Farrell et al., 2011; Farrell et al., 2008; van der Stok et al., 2014), this system was a suitable model for the present study. Several lentiviral controls were included to ensure that transfection of primary MSCs with a lentivirus [empty vector control (SHC001)] and the activation of RNA-induced silencing complex (RISC) [by a non-target control shRNA (SHC002)] was not having detrimental effects on cell behaviour. To ensure that the proper concentration of protamine sulphate, puromycin and lentiviral supernatant were used, extensive testing was completed prior to experimental work start. This allowed to minimise unnecessary stress to MSCs throughout the study. In some instances,

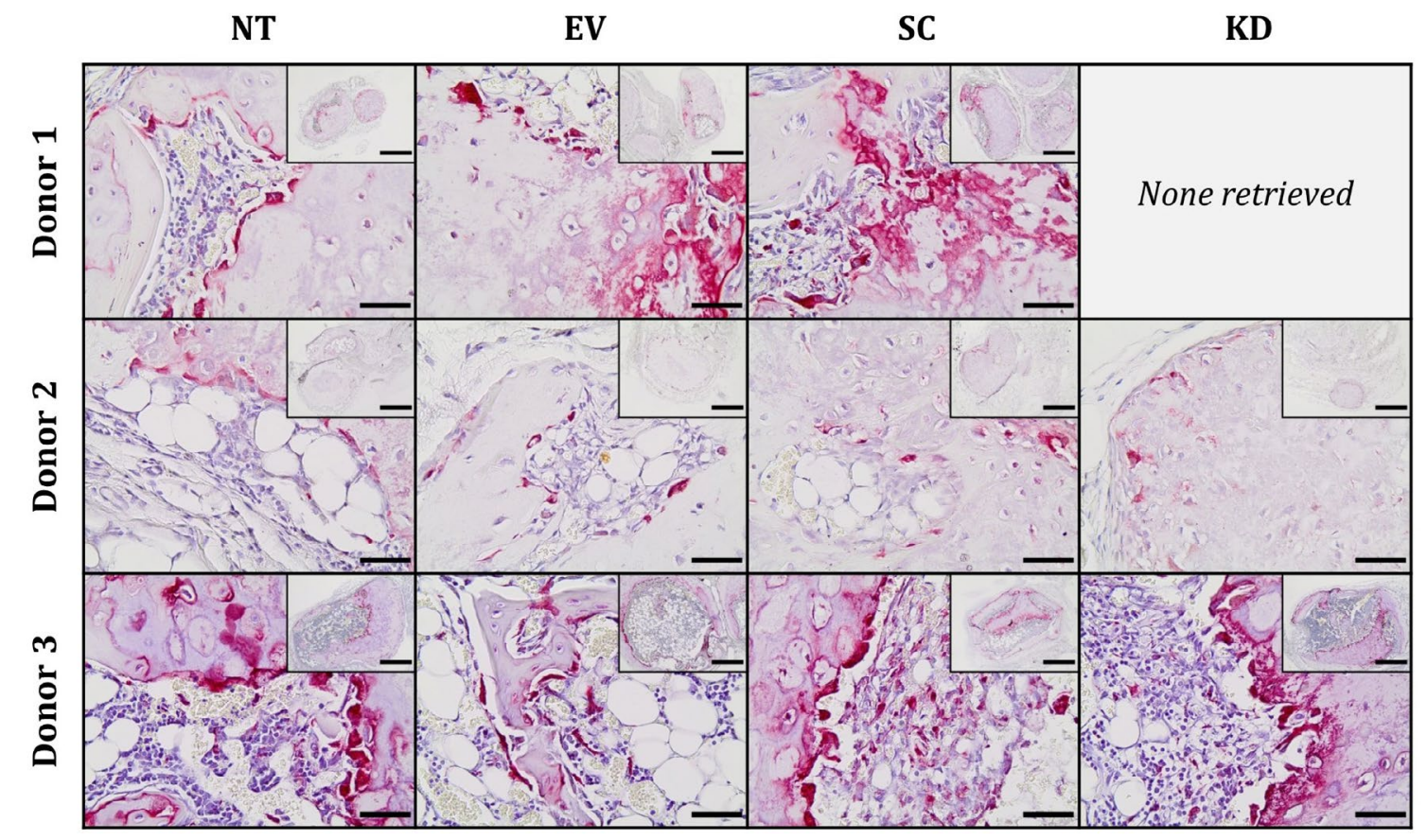

Fig. 6. Osteoclast activity in response to COL10A1 knock-down in chondrogenically differentiated MSCs following in vivo implantation was affected in relation to the efficiency of the knock-down. TRAP staining of implanted pellets following 8 (donors 1 and 2) or 10 (donor 3) weeks of in vivo implantation. Pellets containing COL10A1 shRNA could not be recovered following implantation from donor 1.1 of the 9 COL10A1-shRNA-containing pellets from donor 2 was recovered but only exhibited TRAP positive staining on the outer periphery. All implanted pellets from donor 3 were recovered and displayed TRAP positivity throughout the construct. Staining did not vary greatly among non-treated, empty and nontarget conditions. Scale bar: $200 \mu \mathrm{m}$ (bigger squares) and $50 \mu \mathrm{m}$ (smaller squares). NT: non-treated; EV: empty vector; SC: non-target control; KD: knock-down. 
an increased chondrogenic potential was observed in lentivirally transduced cells (empty vector and non-target vector) as compared to non-treated MSCs. The addition of protamine sulphate could have contributed to this increase, as it has been shown to increase proliferation and differentiation potential when used in transduction protocol (Lin et al., 2012). Protamine sulphate was not included in non-treated control in order to assess the chondrogenic and boneforming potential of truly non-treated cells which had undergone the same number of passages. Given the extensive passaging required to accumulate the number of MSCs required for these experiments and the inherent donor variability observed in MSCs, each donor was shown independently in order to give a clear impression of the results.

Knuth et al. (2017) observed a close relation between COLII and COLX, which are expressed almost simultaneously during early chondrogenic differentiation. It is possible that during chondrogenic differentiation of MSCs, COLX expression is essential for COLII and in the absence of COLX, not only is COLII affected, but also other ECM components which are essential for proper chondrogenic differentiation.
Rosati et al. (1994), in a COL10A1 knock-down and Tg mouse model study, showed that adjacent nonhypertrophic chondrocytes, which are unaffected by the knock-down, are still able to produce normal cartilage matrix components. As these non-affected cells were not present in the present model, this could explain why matrix production was more severely affected. Knuth et al. (2017) and Pelttari et al. (2006) have previously reported that COL10A1 expression occurs early in chondrogenic differentiation of MSCs, being observed within the first few days. In tissue engineering, it appears as if a certain threshold of COL10A1 production is crucial for proper cartilage matrix formation during chondrogenic differentiation. When COL10A1 was efficiently downregulated, proper matrix formation was hindered. Not only were crucial ECM components not produced, but the secreted profile of the constructs was affected. A small quantity of COL10A1 (roughly $20 \%$ of the original; donor 3 ) was sufficient to allow MSC construct to undergo ossification and marrow formation in vivo, albeit less than in non-knock-down conditions. This might indicate that a minimum threshold of COLX production allowed for these a

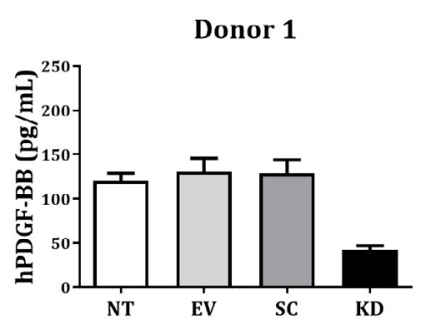

b

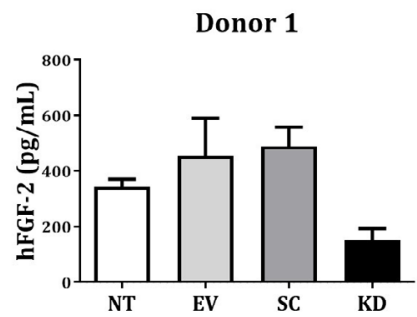

Donor 1

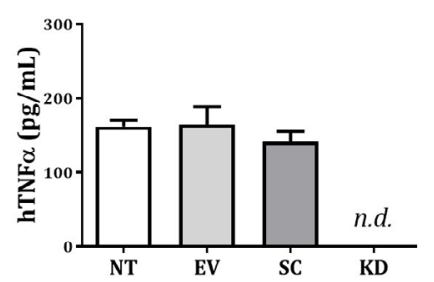

Donor 2

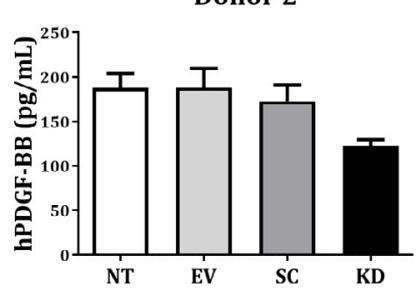

Donor 2

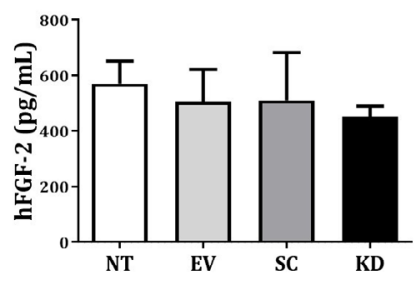

Donor 2

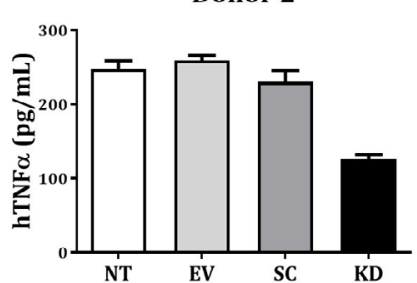

Donor 3

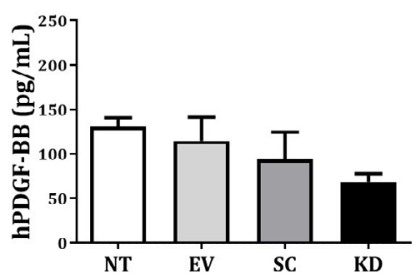

Donor 3

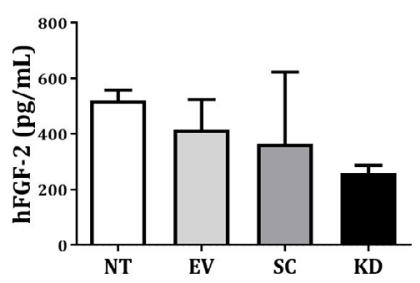

Donor 3

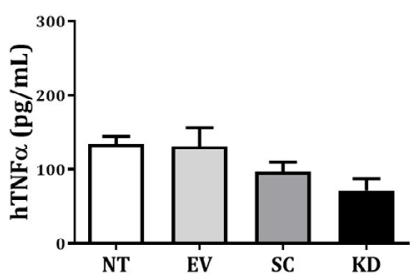

Fig. 7. When COL10A1 production was absent, the production of factors related to osteoclastic and osteoblastic migration and maturation was decreased but a small quantity rescued this effect. (a,b) A significant knock-down of COL10A1 was required for other factors related to osteoblastic migration and maturation, hPDGF-BB and hFGF-2, to be down-regulated (donor 1). A small quantity of COL10A1 remaining in knock-down conditions showed no inhibition of hPDGF-BB and hFGF-2 (donors 2 and 3 ). (c) Production of hTNF $\alpha$ was down regulated when COL10A1 was downregulated in chondrogenic MSCs. Production did not vary greatly between non-treated, empty and non-target conditions. As donor were not pooled, no statistical analysis was performed. NT: non-treated; EV: empty vector; SC: non-target control; KD: knock-down. n.d.: not determined. 
processes to still occur. A larger number of donors would be helpful to determine exactly what this threshold is.

Schmid type metaphyseal chondrodysplasia, a disease resulting in dwarfism, coxa vara and other skeletomuscular defects, is the result of mutated or deficient COLX production (Chan et al., 1998; Mcintosh et al., 1994; Warman et al., 1993). As a significant effect was observed when COLX production was decreased during chondrogenesis, how this affected bone formation following implantation was investigated. A severe decrease in COLX production had not only major consequences on MSC chondrogenic differentiation but also affected endochondral bone formation. When COLX was significantly down regulated, as in donor 1 , bone formation was not observed. However, in donor 2, who also had a highly efficient knock-down, only one pellet from the implant pockets was retrieved, with calcification of the construct but no marrow formation. Overall, this donor appeared to be slightly delayed in bone formation, as controls had very little marrow formation following implantation. It is possible that had the in vivo time frame been increased, marrow formation would have occurred, but this would require verification by further investigation. In donor 3, where knock-down efficiency was under $90 \%$, matrix remodelling and bone formation appeared relatively unaffected; however, mineralisation was still less than in non-treated controls. Due to the inherent variability in these types of experiments, because of inter-donor as well as lentivirus batches variations, it was difficult to make a clear broad statement on the knock-down effect in all donors. However, the knock-down variability enabled the conclusion that some degree of COLX expression was sufficient for bone formation to take place, since bone formation was only inhibited in the samples where COLX was knocked-down completely. It is possible that the greater chondrogenic potential of this donor required a higher lentiviral load in order to completely prevent COL10A1 expression due to the presence of more mRNA per cell. However, a higher virus concentration increases the risks of offtarget effects. It would be of great interest to study if the overexpression of COL10A1 in this model would improve chondrogenic differentiation or increase the secretion of factors which influence in vivo matrix remodelling and bone formation. Alternative knock-down approaches, for example a CRISPR-Cas system using hTERT-immortalised MSCs, would be beneficial to confirm those findings.

To further understand these differences, the secretion of relevant molecules was investigated. During EO, recruitment and invasion of the nearby vasculature, initiation of osteoclast migration, remodelling of the cartilaginous matrix and osteoblastic maturation and bone deposition are all essential. Those processes can be initiated by different secreted factors (Boeuf and Richter, 2010; Scotti et al.,
2013; Scotti et al., 2010; Tonnarelli et al., 2014), which were released or found within the matrix of the implanted chondrogenic pellets. TNF $\alpha$ production was downregulated when COLX expression was decreased. TNF $\alpha$ is produced at high levels by hypertrophic chondrocytes (Kon et al., 2001). TNF $\alpha$ is not only a major inflammatory factor involved in bone fracture healing (Gerstenfeld et al., 2003) but also an important factor in bone formation and metabolism. It is involved in osteoclast differentiation and mediates bone turnover (Bertolini et al., 1986; Kimble et al., 1997). In fracture repair studies, a decreased recruitment of osteoclasts as well as delayed endochondral bone formation are observed in mice with $\mathrm{TNF} \alpha$ signalling deficiency (Gerstenfeld et al., 2003). Other factors, including PDGF-BB and FGF-2, which are expressed consistently during all stages of fracture repair (Bolander, 1992), were also found to be affected by a lack of COLX. PDGF is important for osteoblastic differentiation (Li et al., 2014; Yu et al., 1997) and contributes to the stabilisation of blood vessels by orchestrating migration and assembly of pericytes, which contribute to proper vasculature formation (Caplan and Correa, 2011; Xie et al., 2014). FGFs play a role in $\mathrm{EO}$ and are also important for the proliferation and differentiation of chondrocytes (Kronenberg, 2003; Ornitz and Marie, 2002). Additionally, FGFs play a role in osteoclast formation and maturation (Hurley et al., 1998; Kawaguchi et al., 2000; Nakagawa et al., 1999). A significant decrease in TNF $\alpha$, PDGF$\mathrm{BB}$ and FGF-2 was found when COL10A1 was knocked-down over $99 \%$ as compared to controls which did not show a difference in the production of this or other reported factors (donor 1). In donor 1 COL10A1 knock-down, constructs were never retrieved following implantation. These constructs were most likely resorbed or degraded by the host. However, donors which had a decreased COLX expression but still maintained TNF $\alpha$, PDGF-BB and FGF-2 expression were retrieved and formed bone following implantation (donors 2 and 3). In retrieved pellets from these donors, bone and signs of osteoclastic remodelling were observed, as evident by TRAP positive staining. Possibly, the production of these secreted factors was high enough to initiate the migration of osteoclasts and osteoblasts required to initiate bone formation. A full secreted profile analysis would be helpful to further elucidate the effects of a COL10A1 knock-down on the chondrogenic secretome of differentiated MSCs.

In the present study, significant effects of COLX during both cartilage and bone formation, were observed. However, Rosati et al. (1994) found no major defects or skeletal abnormalities when COLX production is inhibited. This could be explained by the species' differences, as neither mouse cells nor a fully immune competent environment were part of the present study. Additionally, it is possible that, in Rosati et al. (1994) study, other collagens and components in the ECM were able to compensate 
for the absence of COLX by surrounding cells in the growth plate. As the present model was a single-cell culture followed by subcutaneous implantation in a normally non-bony environment, no such cells close to the construct were present to compensate for the lack of COLX. As is often the case with MSC studies, a certain amount of inter- and intra-donor variation was observed. Although inter-donor variation is well documented in MSCs, the intradonor variation observed could be attributed to the random integration nature of lentiviral delivery. This could have been controlled partially for by single-cell expansion, which is extremely difficult to achieve when using a primary MSC cell source. This variation has also been observed by others utilising primary MSCs (Lin et al., 2012). However, it is clear from a tissue engineering perspective that COLX is essential for proper cartilage and bone formation. Its presence is required for proper cartilage differentiation pre-implantation and necessary for subsequent endochondral bone formation. With this in mind, it could be possible to further improve graft performance with chondrogenically differentiated MSCs by over expressing or increasing hypertrophic differentiation pre-implantation, augmenting the production of important factors which are influenced by the presence of COLX. However, further studies are required to prove this. By improving the understanding of how these constructs achieve endochondral bone formation, graft performance and clinical relevance of such constructs will also improve.

\section{Conclusion}

The present study showed how the absence of COLX in MSCs not only hindered the ability of MSCs to undergo chondrogenesis but also affected subsequent endochondral ossification following in vivo implantation. From a tissue engineering perspective, the study improved the understanding of how MSC-mediated endochondral ossification is achieved. Understanding COLX plays a crucial role. The present method of bone formation could further improve graft construction in the future.

\section{Acknowledgments}

The work was supported using imaging equipment provided by the Applied Molecular Imaging Erasmus MC facility.

Authors declare no conflict of interest.

\section{References}

Anderson HC, Garimella R, Tague SE (2005) The role of matrix vesicles in growth plate development and biomineralization. Front Biosci 10: 822-837.
Bahney CS, Hu DP, Taylor AJ, Ferro F, Britz HM, Hallgrimsson B, Johnstone B, Miclau T, Marcucio RS (2014) Stem cell-derived endochondral cartilage stimulates bone healing by tissue transformation. J Bone Miner Res 29: 1269-1282.

Bahney CS, Hu DP, Theodore Miclau Iii RSM (2015) The multifaceted role of the vasculature in endochondral fracture repair. Front Endocrinol 6: 4. DOI: 10.3389/fendo.2015.00004.

Bertolini DR, Nedwin GE, Bringman TS, Smith DD, Mundy GR (1986) Stimulation of bone resorption and inhibition of bone formation in vitro by human tumour necrosis factors. Nature 319: 516-518.

Boeuf S, Richter W (2010) Chondrogenesis of mesenchymal stem cells: role of tissue source and inducing factors. Stem Cell Res Ther 1: 31. DOI: 10.1186/scrt31.

Bolander ME (1992) Regulation of fracture repair by growth factors. Proc Soc Exp Biol Med 200: 165-170.

Bourgine PE, Scotti C, Pigeot S, Tchang LA, Todorov A, Martin I (2014) Osteoinductivity of engineered cartilaginous templates devitalized by inducible apoptosis. Proc Natl Acad Sci U S A 111: 17426-17431.

Campbell MR, Gress CJ, Appleman EH, Jacenko $\mathrm{O}$ (2004) Chicken collagen $X$ regulatory sequences restrict transgene expression to hypertrophic cartilage in mice. Am J Pathol 164: 487-499.

Caplan AI, Correa D (2011) PDGF in bone formation and regeneration: new insights into a novel mechanism involving MSCs. J Orthop Res 29: 17951803.

Cervantes-Diaz F, Contreras P, Marcellini S (2017) Evolutionary origin of endochondral ossification: the transdifferentiation hypothesis. Dev Genes Evol 227: 121-127.

Chan D, Jacenko O (1998) Phenotypic and biochemical consequences of collagen $X$ mutations in mice and humans. Matrix Biology 17: 169-184.

Chan D, Weng YM, Graham HK, Sillence DO, Bateman JF (1998) A nonsense mutation in the carboxyl-terminal domain of type $X$ collagen causes haploinsufficiency in schmid metaphyseal chondrodysplasia. J Clin Invest 101: 1490-1499.

Chen Q, Gibney E, Fitch JM, Linsenmayer C, Schmid TM, Linsenmayer T (1990) Long-range movement and fibril association of type $X$ collagen within embryonic cartilage matrix. Proc Natl Acad Sci 87: 8046-8050.

Chen Q, Linsenmayer C, Gu H, Schmid TM, Linsenmayer TF (1992) Domains of type X collagen: alteration of cartilage matrix by fibril association and proteoglycan accumulation. J Cell Biol 117: 687-694.

Colnot CI, Helms JA (2001) A molecular analysis of matrix remodeling and angiogenesis during long bone development. Mech Dev 100: 245-250.

Cunniffe GM, Vinardell T, Murphy JM, Thompson EM, Matsiko A, O’Brien FJ, Kelly DJ (2015) Porous decellularized tissue engineered hypertrophic cartilage as a scaffold for large bone defect healing. Acta Biomater 23: 82-90. 
Deckers MML, Van Bezooijen RL, Van Der Horst G, Hoogendam J, van der Bent C, Papapoulos SE, Löwik CWGM (2002) Bone morphogenetic proteins stimulate angiogenesis through osteoblast-derived vascular endothelial growth factor A. Endocrinology 143: 1545-1553.

Engsig MT, Chen Q-J, Vu TH, Pedersen A-C, Therkidsen B, Lund LR, Henriksen K, Lenhard T, Foged NT, Werb Z (2000) Matrix metalloproteinase 9 and vascular endothelial growth factor are essential for osteoclast recruitment into developing long bones. J Cell Biol 151: 879-890.

Farrell E, Both SK, Odorfer KI, Koevoet W, Kops N, O’Brien FJ, Baatenburg de Jong RJ, Verhaar JA, Cuijpers V, Jansen J, Erben RG, van Osch GJ (2011) Invivo generation of bone via endochondral ossification by in-vitro chondrogenic priming of adult human and rat mesenchymal stem cells. BMC Musculoskelet Disord 12: 31. DOI: 10.1186/1471-2474-12-31.

Farrell E, van der Jagt OP, Koevoet W, Kops N, Van Manen CJ, Hellingman CA, Jahr H, O'Brien FJ, Verhaar JAN, Weinans H (2008) Chondrogenic priming of human bone marrow stromal cells: a better route to bone repair? Tissue Eng Part C Methods 15: 285-295.

Gerstenfeld LC, Cho TJ, Kon T, Aizawa T, Tsay A, Fitch J, Barnes GL, Graves DT, Einhorn TA (2003) Impaired fracture healing in the absence of TNF-alpha signaling: the role of TNF-alpha in endochondral cartilage resorption. J Bone Miner Res 18: 1584-1592.

Gerstenfeld LC, Cullinane DM, Barnes GL, Graves DT, Einhorn TA (2003) Fracture healing as a postnatal developmental process: molecular, spatial, and temporal aspects of its regulation. J Cell Biochem 88: 873-884.

Hurley M, Lee S, Raisz L, Bernecker P, Lorenzo J (1998) Basic fibroblast growth factor induces osteoclast formation in murine bone marrow cultures. Bone 22: 309-316.

Jacenko O, Chan D, Franklin A, Ito S, Underhill CB, Bateman JF, Campbell MR (2001) A dominant interference collagen $X$ mutation disrupts hypertrophic chondrocyte pericellular matrix and glycosaminoglycan and proteoglycan distribution in transgenic mice. Am J Pathol 159: 2257-2269.

Jacenko O, Roberts DW, Campbell MR, McManus PM, Gress CJ, Tao Z (2002) Linking hematopoiesis to endochondral skeletogenesis through analysis of mice transgenic for collagen X. Am J Pathol 160: 2019-2034.

Kawaguchi H, Chikazu D, Nakamura K, Kumegawa M, Hakeda Y (2000) Direct and indirect actions of fibroblast growth factor 2 on osteoclastic bone resorption in cultures. J Bone Miner Res 15: 466-473.

Kim SJ, Kim SY, Kwon CH, Kim YK (2007) Differential effect of FGF and PDGF on cell proliferation and migration in osteoblastic cells. Growth Factors 25: 77-86.

Kim TK, Sharma B, Williams CG, Ruffner MA, Malik A, McFarland EG, Elisseeff JH (2003) Experimental model for cartilage tissue engineering to regenerate the zonal organization of articular cartilage. Osteoarthritis Cartilage 11: 653-664.

Kimble RB, Bain S, Pacifici R (1997) The functional block of TNF but not of IL-6 prevents bone loss in ovariectomized mice. J Bone Miner Res 12: 935-941.

Kirsch T, Pfäffle M (1992) Selective binding of anchorin CII (annexin V) to type II and X collagen and to chondrocalcin (C-propeptide of type II collagen). Implications for anchoring function between matrix vesicles and matrix proteins. FEBS Lett 310: 143-147.

Knuth CA, Kiernan CH, Palomares Cabeza V, Lehmann J, Witte-Buoma J, ten Berge D, Brama PA, Wolvius EB, Strabbing EM, Koudstaal M (2018) Isolating paediatric mesenchymal stem cells with enhanced expansion and differentiation capabilities. Tissue Eng Part C Methods 24: 313-321.

Knuth CA, Witte-Bouma J, Ridwan Y, Wolvius EB, Farrell E (2017) Mesenchymal stem cell-mediated endochondral ossification utilising micropellets and brief chondrogenic priming. Eur Cell Mater 34: 142161.

Kon T, Cho TJ, Aizawa T, Yamazaki M, Nooh N, Graves D, Gerstenfeld LC, Einhorn TA (2001) expression of osteoprotegerin, receptor activator of NF- $\kappa \mathrm{B}$ ligand (osteoprotegerin ligand) and related proinflammatory cytokines during fracture healing. J Bone Miner Res 16: 1004-1014.

Kronenberg HM (2003) Developmental regulation of the growth plate. Nature 423: 332-336.

Kwan KM, Pang MK, Zhou S, Cowan SK, Kong RY, Pfordte T, Olsen BR, Sillence DO, Tam PP, Cheah KS (1997) Abnormal compartmentalization of cartilage matrix components in mice lacking collagen $X$ : implications for function. J Cell Biol 136: 459-471.

Li A, Xia X, Yeh J, Kua H, Liu H, Mishina Y, Hao A, Li B (2014) PDGF-AA promotes osteogenic differentiation and migration of mesenchymal stem cell by down-regulating PDGFR $\alpha$ and derepressing BMP-Smad1/5/8 signaling. PloS One 9: e113785. DOI: 10.1371/journal.pone.0113785.

Lin P, Lin Y, Lennon DP, Correa D, Schluchter M, Caplan AI (2012) Efficient lentiviral transduction of human mesenchymal stem cells that preserves proliferation and differentiation capabilities. Stem Cells Transl Med 1: 886-897.

Livak KJ, Schmittgen TD (2001) Analysis of relative gene expression data using real-time quantitative PCR and the 2(-delta delta C(T)) method. Methods 25: 402-408.

Luvalle P, Daniels K, Hay ED, Olsen BR (1992) Type $X$ collagen is transcriptionally activated and specifically localized during sternal cartilage maturation. Matrix 12: 404-413.

Mackie E (2003) Osteoblasts: novel roles in orchestration of skeletal architecture. Int J Biochem Cell Biol 35: 1301-1305.

Maes C, Kobayashi T, Selig MK, Torrekens S, Roth SI, Mackem S, Carmeliet G, Kronenberg HM (2010) Osteoblast precursors, but not mature osteoblasts, move into developing and fractured bones along with invading blood vessels. Dev Cell 19: 329-344. 
Mcintosh I, Abbott MH, Warman ML, Olsen BR, Francomano CA (1994) Additional mutations of type X collagen confirm COL10A1 as the Schmid metaphyseal chondrodysplasia locus. Hum Mol Genet 3: 303-307.

Mueller MB, Tuan RS (2008) Functional characterization of hypertrophy in chondrogenesis of human mesenchymal stem cells. Arthritis Rheum 58: 1377-1388.

Nakagawa N, Yasuda H, Yano K, Mochizuki S-i, Kobayashi N, Fujimoto H, Shima N, Morinaga T, Chikazu D, Kawaguchi H (1999) Basic fibroblast growth factor induces osteoclast formation by reciprocally regulating the production of osteoclast differentiation factor and osteoclastogenesis inhibitory factor in mouse osteoblastic cells. Biochem Biophys Res Commun 265: 158-163.

Narcisi R, Cleary MA, Brama PA, Hoogduijn MJ, Tüysüz N, ten Berge D, van Osch GJ (2015) Long-term expansion, enhanced chondrogenic potential, and suppression of endochondral ossification of adult human MSCs via WNT signaling modulation. Stem Cell Reports 4: 459-472.

Ornitz DM (2005) FGF signaling in the developing endochondral skeleton. Cytokine Growth Factor Rev 16: 205-213.

Ornitz DM, Marie PJ (2002) FGF signaling pathways in endochondral and intramembranous bone development and human genetic disease. Genes Dev 16: 1446-1465.

Ortega N, Behonick DJ, Werb Z (2004) Matrix remodeling during endochondral ossification. Trends Cell Biol 14: 86-93.

Pelttari K, Winter A, Steck E, Goetzke K, Hennig T, Ochs BG, Aigner T, Richter W (2006) Premature induction of hypertrophy during in vitro chondrogenesis of human mesenchymal stem cells correlates with calcification and vascular invasion after ectopic transplantation in SCID mice. Arthritis Rheum 54: 3254-3266.

Poole AR, Pidoux I (1989) Immunoelectron microscopic studies of type X collagen in endochondral ossification. J Cell Biol 109: 2547-2554.

Rosati R, Horan GS, Pinero GJ, Garofalo S, Keene DR, Horton WA, Vuorio E, de Crombrugghe B, Behringer RR (1994) Normal long bone growth and development in type X collagen-null mice. Nat Genet 8: 129-135.

Schmid TM, Linsenmayer TF (1985) Immunohistochemical localization of short chain cartilage collagen (type $\mathrm{X}$ ) in avian tissues. J Cell Biol 100: 598-605.

Scotti C, Piccinini E, Takizawa H, Todorov A, Bourgine P, Papadimitropoulos A, Barbero A, Manz MG, Martin I (2013) Engineering of a functional bone organ through endochondral ossification. Proc Natl Acad Sci U S A 110: 3997-4002.

Scotti C, Tonnarelli B, Papadimitropoulos A, Scherberich A, Schaeren S, Schauerte A, Lopez-Rios J, Zeller R, Barbero A, Martin I (2010) Recapitulation of endochondral bone formation using human adult mesenchymal stem cells as a paradigm for developmental engineering. Proc Natl Acad Sci U S A 107: 7251-7256.

Shen G (2005) The role of type X collagen in facilitating and regulating endochondral ossification of articular cartilage. Orthod Craniofac Res 8: 11-17.

Sweeney E, Campbell M, Watkins K, Hunter CA, Jacenko O (2008) Altered endochondral ossification in collagen $X$ mouse models leads to impaired immune responses. Dev Dyn 237: 2693-2704.

Sweeney E, Roberts D, Corbo T, Jacenko O (2010) Congenic mice confirm that collagen $X$ is required for proper hematopoietic development. PloS One 5: e9518. DOI: 10.1371/journal.pone.0009518.

Toben D, Schroeder I, El Khassawna T, Mehta M, Hoffmann JE, Frisch JT, Schell H, Lienau J, Serra A, Radbruch A, Duda GN (2011) Fracture healing is accelerated in the absence of the adaptive immune system. J Bone Miner Res 26: 113-124.

Tonnarelli B, Centola M, Barbero A, Zeller R, Martin I (2014) Re-engineering development to instruct tissue regeneration. Curr Top Dev Biol 108: 319-338.

Touaitahuata H, Cres G, de Rossi S, Vives V, Blangy A (2014) The mineral dissolution function of osteoclasts is dispensable for hypertrophic cartilage degradation during long bone development and growth. Dev Biol 393: 57-70.

van der Stok J, Koolen MKE, Jahr H, Kops N, Waarsing JH, Weinans H, van der Jagt OP (2014) Chondrogenically differentiated mesenchymal stromal cell pellets stimulate endochondral bone regeneration in critical-sized bone defects. Eur Cell Mater 27: 137-148.

Warman ML, Abbott M, Apte SS, Hefferon T, McIntosh I, Cohn DH, Hecht JT, Olsen BR, Francomano CA (1993) A type X collagen mutation causes Schmid metaphyseal chondrodysplasia. Nat Genet 5: 79-82.

Wu LN, Genge BR, Lloyd GC, Wuthier RE (1991) Collagen-binding proteins in collagenase-released matrix vesicles from cartilage. Interaction between matrix vesicle proteins and different types of collagen. J Biol Chem 266: 1195-1203.

Xie H, Cui Z, Wang L, Xia Z, Hu Y, Xian L, Li C, Xie L, Crane J, Wan M (2014) PDGF-BB secreted by preosteoclasts induces angiogenesis during coupling with osteogenesis. Nat Med 20: 1270-1278.

Yang L, Tsang KY, Tang HC, Chan D, Cheah KS (2014) Hypertrophic chondrocytes can become osteoblasts and osteocytes in endochondral bone formation. Proc Natl Acad Sci U S A 111: 12097-12102.

Yu X, Hsieh SC, Bao W, Graves DT (1997) Temporal expression of PDGF receptors and PDGF regulatory effects on osteoblastic cells in mineralizing cultures. Am J Physiol 272: C1709-C1716.

Zhou X, von der Mark K, Henry S, Norton W, Adams H, de Crombrugghe B (2014) Chondrocytes transdifferentiate into osteoblasts in endochondral 
bone during development, postnatal growth and fracture healing in mice. PLoS Genet 10: e1004820. DOI: 10.1371/journal.pgen.1004820.

\section{Discussion with Reviewers}

Daniel Kelly: COLX appeared to be important not only for endochondral ossification, but also for initiation of MSC chondrogenesis. What are the implications of this finding for the cartilage tissue engineering field and the widespread belief that COLX expression is always a 'bad thing' as it suggests hypertrophy? Should we also be viewing COLX as a key early marker of MSC chondrogenesis?

Authors: With regard to MSC-based chondrogenesis, we believe that COLX expression closely mirrors the expression of COLII and, therefore, is expressed quite early in chondrogenesis (Fig.1d day 7 and Knuth et al., 2017). However, it is still not desirable in the context of cartilage tissue engineering as it seems to always precede bone formation. In healthy chondrocytes, COLX expression is not observed and is usually also seen as a marker of negative processes in the joint related to osteoarthritis onset and progression. We believe, it is valid to say that it could be a key early marker of MSC chondrogenesis in the currently available protocols for differentiation in vitro, but with the caveat that this chondrogenesis will most probably lead to endochondral ossification.
Daniel Kelly: For the donors that failed to undergo endochondral ossification in vivo, it is difficult to link this directly to the absence of COLX, as, in general, chondrogenesis and ECM accumulation were also supressed in these groups. In the future, might it be possible to silence COL10A1 later in chondrogenesis to better elucidate its role in hypertrophy and endochondral ossification?

Authors: This would involve generating a lentivirus with an extra inducible cassette that enables overexpression of the shRNA for COL10A1 when a specific antibiotic is added to the culture medium. Theoretically, this would enable the knock-down of the gene from a specific time point or for a desired duration. However, there would be some technical issues to overcome. For example, these systems can be very leaky, leading to some expression of the shRNA even without the addition of the specific inducer. Also, penetration of the antibiotic through the dense matrix of the chondrogenic pellet would have to be assessed and verified. In general, it would be very interesting to assess if there is a stage specific necessity for COL10A1 expression.

Editor's note: The Scientific Editor responsible for this paper was Martin Stoddart. 